\title{
Carcinogenesis of Beta-Propiolactone: A Computational Study
}

\author{
Eva Španinger, Urban Bren * \\ Laboratory of Physical Chemistry and Chemical Thermodynamics, Faculty of Chemistry and Chemical Engineering, University of Maribor, \\ Smetanova ulica 17, SI-2000 Maribor, Slovenia; eva.spaninger@um.si \\ *Correspondence: urban.bren@um.si (U.B.); Tel.: +386-2-2294-421 (U.B.)
}

\section{Table of Contents}

Table S1. The calculated activation parameters for the acylation of methylguanine with BPL in the gas phase and solvated with the SCRF, LD and AMSOL models, lowest vibrational frequencies of reactant states, imaginary vibrational frequencies of transition states, and corresponding distances between the reactive centers.

Table S2. The calculated activation parameters for the alkylation of methyladenine with BPL in the gas phase and solvated with the SCRF, LD and AMSOL models, lowest vibrational frequencies of reactant states, imaginary vibrational frequencies of transition states, and corresponding distances between the reactive centers.

Table S3. The calculated activation parameters for the acylation of methyladenine with BPL in the gas phase and solvated with the SCRF, LD and AMSOL models, lowest vibrational frequencies of reactant states, imaginary vibrational frequencies of transition states, and corresponding distances between the reactive centers. 
Table S4. The calculated activation parameters for the alkylation of methycytosine with BPL in the gas phase and solvated with the SCRF, LD and AMSOL models, lowest vibrational frequencies of reactant states, imaginary vibrational frequencies of transition states, and corresponding distances between the reactive centers.

Table S5. The calculated activation parameters for the acylation of methycytosine with BPL in the gas phase and solvated with the SCRF, LD and AMSOL models, lowest vibrational frequencies of reactant states, imaginary vibrational frequencies of transition states, and corresponding distances between the reactive centers.

Table S6. The calculated activation parameters for the alkylation of methythymine with BPL in the gas phase and solvated with the SCRF, LD and AMSOL models, lowest vibrational frequencies of reactant states, imaginary vibrational frequencies of transition states, and corresponding distances between the reactive centers.

Table S7. The calculated activation parameters for the acylation of methythymine with BPL in the gas phase and solvated with the SCRF and LD and AMSOL models, lowest vibrational frequencies of reactant states, imaginary vibrational frequencies of transition states, and corresponding distances between the reactive centers.

Table S8. The calculated activation parameters for the glutathione alkylation with BPL in the gas phase and solvated with the SCRF and LD models, lowest vibrational frequencies of reactant states, imaginary vibrational frequencies of transition states, and corresponding distances between the reactive centers.

Table S9. The calculated activation parameters for the glutathione acylation with BPL in the gas phase and solvated with the SCRF and LD models, lowest vibrational frequencies of reactant states, imaginary vibrational frequencies of transition states, and corresponding distances between the reactive centers.

Table S10. The comparison of the activation free energies for BPL alkylation of the two thymine tautomeric forms used in our calculations... S13 Table S11. Absolute energies and Cartesian atomic coordinates for the alkylation reaction of methylguanine at the M06-2X/6-311++G(d,p) level

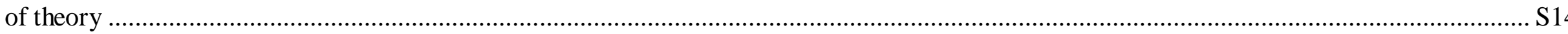
Table S12. Absolute energies and Cartesian atomic coordinates for the acylation reaction of methylguanine at the M06-2X/6-311++G(d,p) level of theory .... ... S17

Table S13. Absolute energies and Cartesian atomic coordinates for the alkylation reaction of methyladenine at the M06-2X/6-311++G(d,p) level

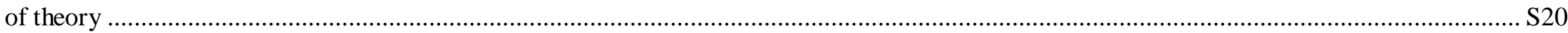
Table S14. Absolute energies and Cartesian atomic coordinates for the acylation reaction of methyladenine at the M06-2X/6-311++G(d,p) level of theory ...... A ... S22

Table S15. Absolute energies and Cartesian atomic coordinates for the alkylation reaction of methylcytosine at the M06-2X/6-311++G(d,p) level of theory 
Table S16. Absolute energies and Cartesian atomic coordinates for the acylation reaction of methylcytosine at the M06-2X/6-311++G(d,p) level of theory ..

Table S17. Absolute energies and Cartesian atomic coordinates for the alkylation reaction of methylthymine lactim tautomeric form at the M06-

$2 \mathrm{X} / 6-311++\mathrm{G}(\mathrm{d}, \mathrm{p})$ level of theory

Table S18. Absolute energies and Cartesian atomic coordinates for the acylation reaction of methylthymine lactim tautomeric form at the M06-

2X/6-311++G(d,p) level of theory S31

Table S19. Absolute energies and Cartesian atomic coordinates for the alkyation reaction of glutathione at the M06-2X/6-311++G(d,p) level of theory $\mathrm{S} 33$

Table S20. Absolute energies and Cartesian atomic coordinates for the acylation reaction of glutathione at the M06-2X/6-311++G(d,p) level of theory.. 
Table S1. The calculated activation parameters for the acylation of methylguanine with BPL in the gas phase and solvated with the SCRF, LD and AMSOL models, lowest vibrational frequencies of reactant states, imaginary vibrational frequencies of transition states, and corresponding distances between the reactive centers.

\begin{tabular}{|c|c|c|c|c|c|c|c|c|c|}
\hline Method/Basis set & $\begin{array}{c}\Delta G_{g a s}^{\ddagger} \\
{[\mathrm{kcal} / \mathrm{mol}]^{\mathrm{a}}}\end{array}$ & $\begin{array}{c}\Delta \Delta G_{\text {hydr }}^{\text {SCRF }} \\
{[\mathrm{kcal} / \mathbf{m o l}]^{b}}\end{array}$ & $\begin{array}{c}\Delta G_{S C R F}^{\ddagger} \\
{[\mathrm{kcal} / \mathrm{mol}]^{\mathrm{c}}}\end{array}$ & $\begin{array}{c}\Delta G_{L D}^{\ddagger} \\
{[\mathrm{kcal} / \mathrm{mol}]^{\mathrm{d}}}\end{array}$ & $\begin{array}{c}\Delta G_{A M S O L}^{\ddagger} \\
{[\mathrm{kcal} / \mathrm{mol}]^{\mathrm{e}}}\end{array}$ & $\begin{array}{c}\omega^{\mathrm{TS}} \\
{\left[\mathrm{i} \mathrm{cm}^{-1}\right]^{\mathrm{f}}}\end{array}$ & $\begin{array}{c}\omega^{\mathrm{R}} \\
{\left[\mathrm{cm}^{-1}\right]^{\mathrm{g}}}\end{array}$ & $\begin{array}{c}\mathbf{d}^{\mathrm{TS}} \\
{[\stackrel{\circ}{\mathrm{A}}]^{\mathrm{h}}}\end{array}$ & $\begin{array}{c}\mathbf{d}^{\mathbf{R}} \\
{\left[\AA{ }^{\mathrm{A}}\right]^{\mathbf{i}}}\end{array}$ \\
\hline AM1 & 46,16 & - & - & - & 26,48 & 772 & 18 & 1,88 & 2,90 \\
\hline PM3 & 36,24 & - & - & - & 22,89 & 357 & 10 & 1,57 & 3,85 \\
\hline $\mathrm{HF} / 6-31 \mathrm{G}(\mathrm{d})$ & 59,20 & $-11,00$ & 48,20 & 41,57 & - & 263 & 19 & 1,56 & 3,06 \\
\hline $\mathrm{HF} / 6-31+\mathrm{G}(\mathrm{d}, \mathrm{p})$ & 58,24 & $-11,87$ & 46,37 & 38,94 & - & 298 & 17 & 1,56 & 3,11 \\
\hline $\mathrm{HF} / 6-311++\mathrm{G}(\mathrm{d}, \mathrm{p})$ & 58,65 & $-11,54$ & 47,11 & 39,96 & - & 309 & 16 & 1,57 & 3,13 \\
\hline B3LYP/6-31 G(d) & 46,70 & $-8,18$ & 38,52 & 34,57 & - & 281 & 20 & 1,57 & 3,01 \\
\hline B3LYP/6-31+ G(d,p) & 45,27 & $-9,50$ & 35,77 & 29,06 & - & 217 & 7 & 1,57 & 3,11 \\
\hline B3LYP/6-311++ G(d,p) & 45,07 & $-9,52$ & 35,55 & 28,83 & - & 188 & 10 & 1,59 & 3,10 \\
\hline MP2/6-31 G(d) & 57,67 & $-19,47$ & 38,20 & 40,37 & - & 389 & 10 & 1,56 & 2,86 \\
\hline MP2/6-31+ G(d,p) & 55,40 & $-22,10$ & 33,30 & 35,04 & - & 272 & 13 & 1,56 & 3,23 \\
\hline MP2/6-311++G(d,p) & 54,62 & $-15,79$ & 38,83 & 34,00 & - & 298 & 18 & 1,56 & 3,24 \\
\hline M06-2X/6-31 G(d) & 48,29 & $-9,43$ & 38,86 & 34,86 & - & 327 & 26 & 1,55 & 2,80 \\
\hline M06-2X/6-31+ G(d,p) & 46,86 & $-10,69$ & 36,17 & 29,84 & - & 320 & 19 & 1,56 & 2,81 \\
\hline M06-2X/6-311++ G(d,p) & 47,32 & $-10,88$ & 36,44 & 29,95 & - & 285 & 20 & 1,57 & 2,82 \\
\hline mPWPW91/6-31 G(d) & 48,08 & $-9,19$ & 38,89 & 34,28 & - & 259 & 20 & 1,53 & 2,93 \\
\hline mPWPW91/6-31+G(d,p) & 46,67 & $-10,17$ & 36,50 & 30,02 & - & 252 & 16 & 1,53 & 2,98 \\
\hline mPWPW91/6-311++ G(d,p) & 46,67 & $-10,31$ & 36,36 & 29,81 & - & 225 & 15 & 1,54 & 2,98 \\
\hline
\end{tabular}

${ }^{a}$ Gas-phase activation energy. ${ }^{b}$ Hydration free energy of the transition state minus hydration free energy of the reactant state obtained by the SCRF method. ${ }^{\mathrm{c}}$ Activation free energy obtained by the SCRF method. ${ }^{\mathrm{d}}$ Activation free energy obtained by the LD method. ${ }^{\mathrm{e}}$ Activation free energy obtained by the AM1-SM1 and PM3 SM3 methods. ${ }^{f}$ The imaginary frequency corresponding to the transition state. ${ }^{g}$ The lowest vibrational frequency corresponding to the reactant state. ${ }^{\mathrm{h}}$ The distance between the reacting N7 atom of methylguanine and the BPL carbonyl carbon atom in the transition state structure. ${ }^{\mathrm{i}}$ The distance between the reacting N7 atom of methylguanine and the BPL carbonyl carbon atom in the reactant state structure. 
Table S2. The calculated activation parameters for the alkylation of methyladenine with BPL in the gas phase and solvated with the SCRF, LD and AMSOL models, lowest vibrational frequencies of reactant states, imaginary vibrational frequencies of transition states, and corresponding distances between the reactive centers.

\begin{tabular}{|c|c|c|c|c|c|c|c|c|c|}
\hline Method/Basis set & $\begin{array}{c}\Delta G_{g a s}^{\ddagger} \\
{[\mathrm{kcal} / \mathrm{mol}]^{\mathrm{a}}}\end{array}$ & $\begin{array}{c}\Delta \Delta G_{h y d r}^{\text {SCRF }} \\
{\left[\mathrm{kcal}^{\mathrm{kgol}}\right]^{\mathrm{b}}}\end{array}$ & $\begin{array}{c}\Delta G_{S C R F}^{\ddagger} \\
{[\mathrm{kcal} / \mathrm{mol}]^{\mathrm{c}}}\end{array}$ & $\begin{array}{c}\Delta G_{L D}^{\ddagger} \\
{[\mathrm{kcal} / \mathrm{mol}]^{\mathrm{d}}}\end{array}$ & $\begin{array}{c}\Delta G_{A M S O L}^{\ddagger} \\
{[\mathrm{kcal} / \mathrm{mol}]^{\mathrm{e}}}\end{array}$ & $\begin{array}{l}\omega^{1 \mathrm{~S}} \\
{\left[\mathrm{i} \mathrm{cm}{ }^{-1}\right]^{\mathrm{f}}}\end{array}$ & $\begin{array}{l}\omega^{\mathrm{R}} \\
{\left[\mathrm{cm}^{-1}\right]^{\mathrm{g}}}\end{array}$ & $\begin{array}{l}d^{1 \mathbf{S}} \\
{[\AA]^{h}}\end{array}$ & 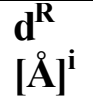 \\
\hline AM1 & 51,68 & - & - & - & 34,92 & 804 & 7 & 1,90 & 3,55 \\
\hline PM3 & 54,29 & - & - & - & 29,95 & 800 & 11 & 1,85 & 3,91 \\
\hline $\mathrm{HF} / 6-31 \mathrm{G}(\mathrm{d})$ & 47,14 & $-17,52$ & 29,62 & 22,71 & - & 619 & 17 & 1,99 & 3,44 \\
\hline $\mathrm{HF} / 6-31+\mathrm{G}(\mathrm{d}, \mathrm{p})$ & 44,72 & $-18,30$ & 26,42 & 19,16 & - & 609 & 18 & 2,02 & 3,49 \\
\hline $\mathrm{HF} / 6-311++\mathrm{G}(\mathrm{d}, \mathrm{p})$ & 45,12 & $-20,72$ & 26,98 & 19,54 & - & 609 & 17 & 2,02 & 3,50 \\
\hline B3LYP/6-31 G(d) & 38,00 & $-15,56$ & 22,44 & 13,88 & - & 487 & 18 & 1,92 & 3,33 \\
\hline B3LYP/6-31+ G(d,p) & 34,50 & $-16,32$ & 18,18 & 8,35 & - & 491 & 12 & 1,96 & 3,46 \\
\hline B3LYP/6-311++ G(d,p) & 34,13 & $-16,16$ & 17,97 & 8,42 & - & 486 & 12 & 1,97 & 3,44 \\
\hline MP2/6-31 G(d) & 46,75 & $-21,07$ & 25,68 & 21,97 & - & 651 & 13 & 2,02 & 3,22 \\
\hline MP2/6-31+ G(d,p) & 44,10 & $-22,57$ & 21,53 & 17,58 & - & 647 & 11 & 2,02 & 3,25 \\
\hline MP2/6-311++G(d,p) & 44,23 & $-21,07$ & 23,16 & 18,29 & - & 670 & 9 & 2,02 & 3,23 \\
\hline M06-2X/6-31 G(d) & 42,26 & $-15,28$ & 26,98 & 19,26 & - & 627 & 27 & 1,87 & 3,13 \\
\hline M06-2X/6-31+ G(d,p) & 39,28 & $-15,74$ & 23,54 & 15,24 & - & 626 & 23 & 1,90 & 3,15 \\
\hline M06-2X/6-311++ G(d,p) & 39,08 & $-15,75$ & 23,33 & 15,01 & - & 633 & 8 & 1,91 & 3,16 \\
\hline mPWPW91/6-31 G(d) & 41,10 & $-15,50$ & 25,60 & 18,07 & - & 609 & 19 & 1,89 & 3,27 \\
\hline mPWPW91/6-31+G(d,p) & 38,43 & $-15,90$ & 22,53 & 13,71 & - & 615 & 9 & 1,92 & 3,36 \\
\hline mPWPW91/6-311++ G(d,p) & 38,27 & $-15,74$ & 22,53 & 14,00 & - & 615 & 10 & 1,92 & 3,36 \\
\hline
\end{tabular}

a Gas-phase activation energy. ${ }^{b}$ Hydration free energy of the transition state minus hydration free energy of the reactant state obtained by the SCRF method. ${ }^{c}$ Activation free energy obtained by the SCRF method. ${ }^{\mathrm{d}}$ Activation free energy obtained by the LD method. ${ }^{\mathrm{e}}$ Activation free energy obtained by the AM1-SM1 and PM3-SM3 methods. ${ }^{f}$ The imaginary frequency corresponding to the transition state. ${ }^{g}$ The lowest vibrational frequency corresponding to the reactant state. ${ }^{\mathrm{h}}$ The distance between the reacting N1 atom of methyladenine and the BPL $\beta$ carbon atom in the transition state structure. ${ }^{\mathrm{i}}$ The distance between the reacting N1 atom of methyladenine and the BPL $\beta$ carbon atom in the reactant state structure. 
Table S3. The calculated activation parameters for the acylation of methyladenine with BPL in the gas phase and solvated with the SCRF, LD and AMSOL models, lowest vibrational frequencies of reactant states, imaginary vibrational frequencies of transition states, and corresponding distances between the reactive centers.

\begin{tabular}{|c|c|c|c|c|c|c|c|c|c|}
\hline Method/Basis set & $\begin{array}{c}\Delta G_{\text {gas }}^{\ddagger} \\
{[\mathrm{kcal} / \mathrm{mol}]^{\mathrm{a}}}\end{array}$ & $\begin{array}{c}\Delta \Delta G_{h y d r}^{\text {SCRF }} \\
{[\mathrm{kcal} / \mathrm{mol}]^{b}}\end{array}$ & $\begin{array}{c}\Delta G_{S C R F}^{\ddagger} \\
{[\mathrm{kcal} / \mathrm{mol}]^{\mathrm{c}}}\end{array}$ & $\begin{array}{c}\Delta G_{L D}^{\ddagger} \\
{[\mathrm{kcal} / \mathrm{mol}]^{\mathrm{d}}}\end{array}$ & $\begin{array}{c}\Delta G_{A M S O L}^{\ddagger} \\
{[\mathrm{kcal} / \mathrm{mol}]^{\mathrm{e}}}\end{array}$ & $\begin{array}{l}\omega^{\mathrm{IS}} \\
{\left[\mathrm{i} \mathrm{cm}^{-1}\right]^{\mathrm{f}}}\end{array}$ & $\begin{array}{l}\omega^{\mathrm{R}} \\
{\left[\mathrm{cm}^{-1}\right]^{\mathrm{g}}}\end{array}$ & $\begin{array}{l}d^{\text {IS }} \\
{[\AA]^{h}}\end{array}$ & 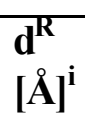 \\
\hline AM1 & 41,41 & - & - & 44,96 & 31,28 & 347 & 7 & 1,51 & $\overline{3,86}$ \\
\hline PM3 & 35,91 & - & - & 45,84 & 30,95 & 342 & 11 & 1,58 & 3,98 \\
\hline $\mathrm{HF} / 6-31 \mathrm{G}(\mathrm{d})$ & 53,77 & $-4,39$ & 49,38 & 45,93 & - & 360 & 17 & 1,58 & 3,56 \\
\hline $\mathrm{HF} / 6-31+\mathrm{G}(\mathrm{d}, \mathrm{p})$ & 52,99 & $-4,54$ & 48,45 & 30,05 & - & 387 & 18 & 1,58 & 3,74 \\
\hline $\mathrm{HF} / 6-311++\mathrm{G}(\mathrm{d}, \mathrm{p})$ & 53,23 & $-4,57$ & 48,66 & 29,20 & - & 372 & 17 & 1,58 & 3,73 \\
\hline B3LYP/6-31 G(d) & 33,22 & $-3,07$ & 30,15 & 29,72 & - & 498 & 18 & 1,63 & 3,69 \\
\hline B3LYP/6-31+ G(d,p) & 33,06 & $-3,01$ & 30,05 & 50,31 & - & 457 & 12 & 1,63 & 4,36 \\
\hline B3LYP/6-311++ G(d,p) & 33,57 & $-2,98$ & 30,59 & 48,35 & - & 453 & 12 & 1,65 & 4,35 \\
\hline MP2/6-31 G(d) & 52,54 & $-23,66$ & 28,88 & 49,62 & - & 793 & 13 & 1,63 & 3,02 \\
\hline MP2/6-31+ G(d,p) & 50,96 & $-24,05$ & 26,91 & 30,43 & - & 663 & 11 & 1,61 & 3,07 \\
\hline MP2/6-311++G(d,p) & 51,84 & $-24,37$ & 27,47 & 28,40 & - & 681 & 9 & 1,61 & 3,02 \\
\hline $\mathrm{M} 06-2 \mathrm{X} / 6-31 \mathrm{G}(\mathrm{d})$ & 32,79 & $-3,22$ & 29,57 & 29,23 & - & 711 & 27 & 1,63 & 3,05 \\
\hline M06-2X/6-31+ G(d,p) & 31,60 & $-3,55$ & 28,05 & 29,95 & - & 699 & 23 & 1,63 & 3,05 \\
\hline M06-2X/6-311++ G(d,p) & 32,30 & $-3,61$ & 28,69 & 28,33 & - & 692 & 8 & 1,64 & 3,03 \\
\hline mPWPW91/6-31 G(d) & 33,93 & $-3,54$ & 30,39 & 29,24 & - & 701 & 19 & 1,56 & 3,56 \\
\hline mPWPW91/6-31+G(d,p) & 32,75 & $-3,50$ & 29,25 & 44,96 & - & 720 & 9 & 1,56 & 4,04 \\
\hline mPWPW91/6-311++ G(d,p) & 33,39 & $-3,51$ & 29,88 & 45,84 & - & 681 & 10 & 1,57 & 3,99 \\
\hline
\end{tabular}

a Gas-phase activation energy. ${ }^{b}$ Hydration free energy of the transition state minus hydration free energy of the reactant state obtained by the SCRF method. ${ }^{c}$ Activation free energy obtained by the SCRF method. ${ }^{\mathrm{d}}$ Activation free energy obtained by the LD method. ${ }^{\mathrm{e}}$ Activation free energy obtained by the AM1-SM1 and PM3-SM3 methods. ${ }^{f}$ The imaginary frequency corresponding to the transition state. ${ }^{g}$ The lowest vibrational frequency corresponding to the reactant state. ${ }^{\mathrm{h}}$ The distance between the reacting N1 atom of methyladenine and the BPL carbonyl carbon atom in the transition state structure. ${ }^{\mathrm{i}}$ The distance between the reacting N1 atom of methyladenine and the BPL carbonyl carbon atom in the reactant state structure. 
Table S4. The calculated activation parameters for the alkylation of methycytosine with BPL in the gas phase and solvated with the SCRF, LD and AMSOL models, lowest vibrational frequencies of reactant states, imaginary vibrational frequencies of transition states, and corresponding distances between the reactive centers.

\begin{tabular}{|c|c|c|c|c|c|c|c|c|c|}
\hline Method/Basis set & $\begin{array}{c}\Delta G_{\text {gas }}^{\ddagger} \\
{[\mathrm{kcal} / \mathrm{mol}]^{\mathrm{a}}}\end{array}$ & $\begin{array}{c}\Delta \Delta G_{h y d r}^{S C R F} \\
{[\mathrm{kcal} / \mathrm{mol}]^{\mathrm{b}}}\end{array}$ & $\begin{array}{c}\Delta G_{S C R F}^{\ddagger} \\
{[\mathrm{kcal} / \mathrm{mol}]^{\mathrm{c}}}\end{array}$ & $\begin{array}{c}\Delta G_{L D}^{\ddagger} \\
{[\mathrm{kcal} / \mathrm{mol}]^{\mathrm{d}}}\end{array}$ & $\begin{array}{c}\Delta G_{A M S O L}^{\ddagger} \\
{[\mathrm{kcal} / \mathrm{mol}]^{\mathrm{e}}}\end{array}$ & $\begin{array}{l}\omega^{\mathrm{TS}} \\
{\left[\mathrm{i} \mathbf{~ c m}^{-1}\right]^{\mathrm{f}}}\end{array}$ & $\begin{array}{l}\omega^{\mathrm{R}} \\
{\left[\mathrm{cm}^{-1}\right]^{\mathrm{g}}}\end{array}$ & $\begin{array}{l}d^{\text {IS }} \\
{\left[\AA^{\AA}\right]^{h}}\end{array}$ & $\begin{array}{l}d^{R} \\
{[\AA]^{i}}\end{array}$ \\
\hline AM1 & 52,98 & - & - & - & 36,67 & 778 & 19 & 1,92 & 6,77 \\
\hline PM3 & 56,03 & - & - & - & 19,13 & 784 & 13 & 1,85 & 4,62 \\
\hline $\mathrm{HF} / 6-31 \mathrm{G}(\mathrm{d})$ & 49,18 & $-21,44$ & 27,74 & 19,29 & - & 576 & 18 & 1,99 & 3,62 \\
\hline $\mathrm{HF} / 6-31+\mathrm{G}(\mathrm{d}, \mathrm{p})$ & 46,84 & $-22,31$ & 24,53 & 13,14 & - & 561 & 28 & 2,02 & 3,66 \\
\hline $\mathrm{HF} / 6-311++\mathrm{G}(\mathrm{d}, \mathrm{p})$ & 47,11 & $-21,96$ & 25,15 & 13,87 & - & 562 & 29 & 2,03 & 3,69 \\
\hline B3LYP/6-31 G(d) & 41,06 & $-19,56$ & 21,50 & 10,84 & - & 486 & 29 & 1,91 & 3,55 \\
\hline B3LYP/6-31+ G(d,p) & 37,37 & $-20,40$ & 16,97 & 4,22 & - & 487 & 26 & 1,95 & 3,61 \\
\hline B3LYP/6-311++ G(d,p) & 36,79 & $-20,13$ & 16,66 & 3,86 & - & 481 & 29 & 1,96 & 3,62 \\
\hline MP2/6-31 G(d) & 49,74 & $-25,27$ & 24,47 & 17,09 & - & 653 & 11 & 1,88 & 3,43 \\
\hline MP2/6-31+ G(d,p) & 47,29 & $-27,21$ & 20,08 & 12,36 & - & 650 & 21 & 1,90 & 3,41 \\
\hline MP2/6-311++G(d,p) & 47,88 & $-26,12$ & 21,76 & 13,73 & - & 673 & 21 & 1,90 & 3,40 \\
\hline M06-2X/6-31 G(d) & 45,74 & $-18,98$ & 26,76 & 17,46 & - & 624 & 27 & 1,88 & 3,20 \\
\hline M06-2X/6-31+ G(d,p) & 42,94 & $-19,76$ & 23,18 & 12,37 & - & 629 & 32 & 1,91 & 3,23 \\
\hline M06-2X/6-311++ G(d,p) & 42,58 & $-19,50$ & 23,08 & 12,53 & - & 638 & 28 & 1,91 & 3,25 \\
\hline mPWPW91/6-31 G(d) & 44,15 & $-19,46$ & 24,69 & 15,25 & - & 601 & 13 & 1,90 & 3,47 \\
\hline mPWPW91/6-31+G(d,p) & 41,65 & $-20,21$ & 21,44 & 10,49 & - & 605 & 22 & 1,92 & 3,50 \\
\hline mPWPW91/6-311++ G(d,p) & 41,37 & $-19,94$ & 21,43 & 10,31 & - & 606 & 21 & 1,93 & 3,52 \\
\hline
\end{tabular}

a Gas-phase activation energy. ${ }^{b}$ Hydration free energy of the transition state minus hydration free energy of the reactant state obtained by the SCRF method. ${ }^{\mathrm{d}}$ Activation free energy obtained by the SCRF method. ${ }^{\mathrm{d}}$ Activation free energy obtained by the LD method. ${ }^{\mathrm{e}}$ Activation free energy obtained by the AM1-SM1 and PM3-SM3 methods. ${ }^{f}$ The imaginary frequency corresponding to the transition state. ${ }^{g}$ The lowest vibrational frequency corresponding to the reactant state. ${ }^{\mathrm{h}}$ The distance between the reacting N3 atom of methylcytosine and the BPL $\beta$ carbon atom in the transition state structure. ${ }^{i}$ The distance between the reacting N3 atom of methylcytosine and the BPL $\beta$ carbon atom in the reactant state structure. 
Table S5. The calculated activation parameters for the acylation of methycytosine with BPL in the gas phase and solvated with the SCRF, LD and AMSOL models, lowest vibrational frequencies of reactant states, imaginary vibrational frequencies of transition states, and corresponding distances between the reactive centers.

\begin{tabular}{|c|c|c|c|c|c|c|c|c|c|}
\hline Method/Basis set & $\begin{array}{c}\Delta G_{\text {gas }}^{\ddagger} \\
{[\mathrm{kcal} / \mathrm{mol}]^{\mathrm{a}}}\end{array}$ & $\begin{array}{c}\Delta \Delta G_{h y d r}^{\text {SCRF }} \\
{[\mathrm{kcal} / \mathrm{mol}]^{\mathrm{b}}}\end{array}$ & $\begin{array}{c}\Delta G_{S C R F}^{\ddagger} \\
{[\mathrm{kcal} / \mathrm{mol}]^{\mathrm{c}}}\end{array}$ & $\begin{array}{c}\Delta G_{L D}^{\ddagger} \\
{[\mathrm{kcal} / \mathrm{mol}]^{\mathrm{d}}}\end{array}$ & $\begin{array}{c}\Delta G_{A M S O L}^{\ddagger} \\
{[\mathrm{kcal} / \mathrm{mol}]^{\mathrm{e}}}\end{array}$ & $\begin{array}{l}\omega^{\mathrm{TS}} \\
{\left[\mathrm{i} \mathbf{~ c m}^{-1}\right]^{\mathrm{f}}}\end{array}$ & $\begin{array}{l}\omega^{\mathrm{R}} \\
{\left[\mathrm{cm}^{-1}\right]^{\mathrm{g}}}\end{array}$ & $\begin{array}{l}d^{\text {IS }} \\
{\left[\AA^{\AA}\right]^{h}}\end{array}$ & $\begin{array}{l}d^{R} \\
{[\AA]^{i}}\end{array}$ \\
\hline AM1 & 39,92 & - & - & - & 15,99 & 259 & 24 & 1,51 & 2,87 \\
\hline PM3 & 35,68 & - & - & - & 18,60 & 361 & 13 & 1,62 & 3,72 \\
\hline $\mathrm{HF} / 6-31 \mathrm{G}(\mathrm{d})$ & 55,28 & $-6,53$ & 48,75 & 44,59 & - & 458 & 18 & 1,69 & 3,16 \\
\hline $\mathrm{HF} / 6-31+\mathrm{G}(\mathrm{d}, \mathrm{p})$ & 54,85 & $-6,82$ & 48,03 & 43,14 & - & 479 & 28 & 1,67 & 3,18 \\
\hline $\mathrm{HF} / 6-311++\mathrm{G}(\mathrm{d}, \mathrm{p})$ & 54,88 & $-6,28$ & 48,60 & 44,49 & - & 480 & 29 & 1,71 & 3,19 \\
\hline B3LYP/6-31 G(d) & 35,31 & $-4,48$ & 30,83 & 30,59 & - & 763 & 29 & 1,78 & 3,12 \\
\hline B3LYP/6-31+ G(d,p) & 34,87 & $-4,51$ & 30,36 & 29,11 & - & 647 & 26 & 1,78 & 3,15 \\
\hline B3LYP/6-311++ G(d,p) & 34,52 & $-4,22$ & 30,30 & 29,77 & - & 685 & 29 & 1,82 & 3,15 \\
\hline $\mathrm{MP} 2 / 6-31 \mathrm{G}(\mathrm{d})$ & 57,25 & $-28,95$ & 28,30 & 51,87 & - & 973 & 11 & 1,79 & 2,99 \\
\hline MP2/6-31+ G(d,p) & 55,73 & $-30,10$ & 25,63 & 54,72 & - & 814 & 21 & 1,78 & 2,97 \\
\hline MP2/6-311++G(d,p) & 56,35 & $-30,47$ & 25,88 & 51,76 & - & 868 & 21 & 1,83 & 2,97 \\
\hline M06-2X/6-31 G(d) & 34,56 & $-4,59$ & 29,97 & 29,23 & - & 737 & 27 & 1,76 & 2,86 \\
\hline M06-2X/6-31+ G(d,p) & 33,75 & $-5,10$ & 28,65 & 27,42 & - & 724 & 32 & 1,75 & 2,90 \\
\hline M06-2X/6-311++ G(d,p) & 33,91 & $-4,45$ & 29,46 & 29,10 & - & 758 & 28 & 1,79 & 2,90 \\
\hline mPWPW91/6-31 G(d) & 37,47 & $-5,91$ & 31,56 & 29,68 & - & 762 & 13 & 1,68 & 3,04 \\
\hline mPWPW91/6-31+G(d,p) & 36,42 & $-6,22$ & 30,20 & 27,48 & - & 704 & 22 & 1,66 & 3,05 \\
\hline mPWPW91/6-311++ G(d,p) & 36,45 & $-5,94$ & 30,51 & 27,91 & - & 694 & 21 & 1,68 & 3,06 \\
\hline
\end{tabular}

a Gas-phase activation energy. ${ }^{b}$ Hydration free energy of the transition state minus hydration free energy of the reactant state obtained by the SCRF method. ${ }^{c}$ Activation free energy obtained by the SCRF method. ${ }^{\mathrm{d}}$ Activation free energy obtained by the LD method. ${ }^{\mathrm{e}}$ Activation free energy obtained by the AM1-SM1 and PM3-SM3 methods. ${ }^{f}$ The imaginary frequency corresponding to the transition state. ${ }^{g}$ The lowest vibrational frequency corresponding to the reactant state. ${ }^{\mathrm{h}}$ The distance between the reacting N3 atom of methylcytosine and the BPL carbonyl carbon atom in the transition state structure. ${ }^{\mathrm{i}}$ The distance between the reacting N3 atom of methylcytosine and the BPL carbonyl carbon atom in the reactant state structure. 
Table S6. The calculated activation parameters for the alkylation of methythymine with BPL in the gas phase and solvated with the SCRF, LD and AMSOL models, lowest vibrational frequencies of reactant states, imaginary vibrational frequencies of transition states, and corresponding distances between the reactive centers.

\begin{tabular}{|c|c|c|c|c|c|c|c|c|c|}
\hline Method/Basis set & $\begin{array}{c}\Delta G_{\text {gas }}^{\ddagger} \\
{[\mathrm{kcal} / \mathrm{mol}]^{\mathrm{a}}}\end{array}$ & $\begin{array}{c}\Delta \Delta G_{h y d r}^{\text {SCRF }} \\
{[\mathrm{kcal} / \mathrm{mol}]^{\mathrm{b}}}\end{array}$ & $\begin{array}{c}\Delta G_{S C R F}^{\ddagger} \\
{[\mathrm{kcal} / \mathrm{mol}]^{\mathrm{c}}}\end{array}$ & $\begin{array}{c}\Delta G_{L D}^{\ddagger} \\
{[\mathrm{kcal} / \mathrm{mol}]^{\mathrm{d}}}\end{array}$ & $\begin{array}{c}\Delta G_{A M S O L}^{\ddagger} \\
{[\mathrm{kcal} / \mathrm{mol}]^{\mathrm{e}}}\end{array}$ & $\begin{array}{l}\omega^{\mathrm{TS}} \\
{\left[\mathrm{i} \mathbf{~ c m}^{-1}\right]^{\mathrm{f}}}\end{array}$ & $\begin{array}{l}\omega^{\mathrm{R}} \\
{\left[\mathrm{cm}^{-1}\right]^{\mathrm{g}}}\end{array}$ & $\begin{array}{l}d^{\text {IS }} \\
{\left[\AA^{\AA}\right]^{h}}\end{array}$ & $\begin{array}{l}d^{R} \\
{[\AA]^{i}}\end{array}$ \\
\hline AM1 & 66,96 & - & - & - & 29,76 & 788 & 13 & 1,89 & $\overline{4,43}$ \\
\hline РM3 & 94,60 & - & - & - & 58,17 & 773 & 18 & 1,85 & 4,75 \\
\hline $\mathrm{HF} / 6-31 \mathrm{G}(\mathrm{d})$ & 71,71 & $-24,19$ & 47,52 & 41,51 & - & 560 & 28 & 1,97 & 3,63 \\
\hline $\mathrm{HF} / 6-31+\mathrm{G}(\mathrm{d}, \mathrm{p})$ & 66,91 & $-25,09$ & 41,82 & 34,01 & - & 543 & 28 & 2,00 & 3,67 \\
\hline $\mathrm{HF} / 6-311++\mathrm{G}(\mathrm{d}, \mathrm{p})$ & 67,16 & $-24,55$ & 42,61 & 34,25 & - & 542 & 29 & 2,01 & 3,70 \\
\hline B3LYP/6-31 G(d) & 60,38 & $-20,67$ & 39,71 & 31,57 & - & 479 & 18 & 1,89 & 3,54 \\
\hline B3LYP/6-31+ G(d,p) & 55,01 & $-21,84$ & 33,17 & 22,46 & - & 478 & 24 & 1,89 & 3,63 \\
\hline B3LYP/6-311++ G(d,p) & 54,71 & $-21,52$ & 33,19 & 22,98 & - & 473 & 24 & 1,94 & 3,64 \\
\hline $\mathrm{MP} 2 / 6-31 \mathrm{G}(\mathrm{d})$ & 72,23 & $-29,91$ & 42,32 & 40,25 & - & 648 & 26 & 1,97 & 3,40 \\
\hline MP2/6-31+ G(d,p) & 66,98 & $-32,07$ & 34,91 & 33,09 & - & 647 & 29 & 1,85 & 3,39 \\
\hline MP2/6-311++G(d,p) & 67,61 & $-31,77$ & 35,84 & 34,69 & - & 670 & 29 & 1,88 & 3,39 \\
\hline M06-2X/6-31 G(d) & 64,34 & $-20,83$ & 43,51 & 37,05 & - & 634 & 30 & 1,86 & 3,28 \\
\hline M06-2X/6-31+ G(d,p) & 59,54 & $-21,58$ & 37,96 & 29,66 & - & 641 & 25 & 1,89 & 3,33 \\
\hline M06-2X/6-311++ G(d,p) & 59,58 & $-21,23$ & 38,35 & 28,62 & - & 648 & 24 & 1,90 & 3,37 \\
\hline mPWPW91/6-31 G(d) & 64,52 & $-19,75$ & 44,77 & 36,33 & - & 596 & 26 & 1,87 & 3,46 \\
\hline mPWPW91/6-31+G(d,p) & 59,89 & $-20,56$ & 39,33 & 29,05 & - & 600 & 23 & 1,90 & 3,50 \\
\hline mPWPW91/6-311++ G(d,p) & 59,76 & $-20,15$ & 39,61 & 27,97 & - & 601 & 23 & 1,91 & 3,53 \\
\hline
\end{tabular}

a Gas-phase activation energy. ${ }^{b}$ Hydration free energy of the transition state minus hydration free energy of the reactant state obtained by the SCRF method. ${ }^{c}$ Activation free energy obtained by the SCRF method. ${ }^{\mathrm{d}}$ Activation free energy obtained by the LD method. ${ }^{\mathrm{e}}$ Activation free energy obtained by the AM1-SM1 and PM3-SM3 methods. ${ }^{f}$ The imaginary frequency corresponding to the transition state. ${ }^{g}$ The lowest vibrational frequency corresponding to the reactant state. ${ }^{\mathrm{h}}$ The distance between the reacting N3 atom of methythymine and the BPL $\beta$ carbon atom in the transition state structure. ${ }^{\mathrm{i}}$ The distance between the reacting N3 atom of methythymine and the BPL $\beta$ carbon atom in the reactant state structure. 
Table S7. The calculated activation parameters for the acylation of methythymine with BPL in the gas phase and solvated with the SCRF and LD and AMSOL models, lowest vibrational frequencies of reactant states, imaginary vibrational frequencies of transition states, and corresponding distances between the reactive centers.

\begin{tabular}{|c|c|c|c|c|c|c|c|c|c|}
\hline Method/Basis set & $\begin{array}{c}\Delta G_{\text {gas }}^{\ddagger} \\
{[\mathrm{kcal} / \mathrm{mol}]^{\mathrm{a}}}\end{array}$ & $\begin{array}{c}\Delta \Delta G_{h y d r}^{\text {SCRF }} \\
{[\mathrm{kcal} / \mathrm{mol}]^{\mathrm{b}}}\end{array}$ & $\begin{array}{c}\Delta G_{S C R F}^{\ddagger} \\
{[\mathrm{kcal} / \mathrm{mol}]^{\mathrm{c}}}\end{array}$ & $\begin{array}{c}\Delta G_{L D}^{\ddagger} \\
{[\mathrm{kcal} / \mathrm{mol}]^{\mathrm{d}}}\end{array}$ & $\begin{array}{c}\Delta G_{A M S O L}^{\ddagger} \\
{[\mathrm{kcal} / \mathrm{mol}]^{\mathrm{e}}}\end{array}$ & $\begin{array}{l}\omega^{\mathrm{TS}} \\
{\left[\mathrm{i} \mathbf{~ c m}^{-1}\right]^{\mathrm{f}}}\end{array}$ & $\begin{array}{l}\omega^{\mathrm{R}} \\
{\left[\mathrm{cm}^{-1}\right]^{\mathrm{g}}}\end{array}$ & $\begin{array}{l}d^{\text {IS }} \\
{\left[\AA^{\AA}\right]^{h}}\end{array}$ & $\begin{array}{l}d^{R} \\
{[\AA]^{i}}\end{array}$ \\
\hline AM1 & 75,32 & - & - & - & 41,81 & 314 & 13 & 1,53 & 3,20 \\
\hline PM3 & 96,20 & - & - & - & 78,08 & 471 & 18 & 1,59 & 3,47 \\
\hline $\mathrm{HF} / 6-31 \mathrm{G}(\mathrm{d})$ & 104,01 & $-16,41$ & 87,60 & 83,93 & - & 560 & 28 & 1,57 & 3,02 \\
\hline $\mathrm{HF} / 6-31+\mathrm{G}(\mathrm{d}, \mathrm{p})$ & 100,53 & $-18,06$ & 82,47 & 76,63 & - & 289 & 28 & 1,56 & 3,05 \\
\hline $\mathrm{HF} / 6-311++\mathrm{G}(\mathrm{d}, \mathrm{p})$ & 100,17 & $-17,71$ & 82,46 & 76,27 & - & 298 & 29 & 1,57 & 3,06 \\
\hline B3LYP/6-31 G(d) & 82,38 & $-9,34$ & 73,04 & 70,31 & - & 304 & 18 & 1,60 & 2,96 \\
\hline B3LYP/6-31+ G(d,p) & 80,18 & $-10,64$ & 69,54 & 64,78 & - & 272 & 24 & 1,59 & 3,00 \\
\hline B3LYP/6-311++ G(d,p) & 79,80 & $-10,76$ & 69,04 & 64,26 & - & 253 & 24 & 1,60 & 3,02 \\
\hline $\mathrm{MP} 2 / 6-31 \mathrm{G}(\mathrm{d})$ & 108,61 & $-39,47$ & 69,14 & 92,31 & - & 385 & 26 & 1,61 & 2,85 \\
\hline MP2/6-31+ G(d,p) & 104,82 & $-42,60$ & 62,22 & 86,21 & - & 363 & 29 & 1,61 & 2,86 \\
\hline MP2/6-311++G(d,p) & 104,66 & $-42,88$ & 61,78 & 86,46 & - & 379 & 29 & 1,59 & 2,86 \\
\hline M06-2X/6-31 G(d) & 80,98 & $-10,34$ & 70,64 & 68,70 & - & 371 & 30 & 1,59 & 2,79 \\
\hline M06-2X/6-31+ G(d,p) & 78,45 & $-11,21$ & 67,24 & 63,67 & - & 359 & 25 & 1,58 & 2,83 \\
\hline M06-2X/6-311++ G(d,p) & 78,64 & $-11,45$ & 67,19 & 62,45 & - & 346 & 24 & 1,59 & 2,83 \\
\hline mPWPW91/6-31 G(d) & 85,50 & $-9,28$ & 76,22 & 72,65 & - & 354 & 26 & 1,55 & 2,87 \\
\hline mPWPW91/6-31+G(d,p) & 82,85 & $-10,12$ & 72,73 & 67,09 & - & 342 & 23 & 1,54 & 2,90 \\
\hline mPWPW91/6-311++ G(d,p) & 82,62 & $-10,32$ & 72,30 & 65,07 & - & 308 & 23 & 1,55 & 2,91 \\
\hline
\end{tabular}

a Gas-phase activation energy. ${ }^{b}$ Hydration free energy of the transition state minus hydration free energy of the reactant state obtained by the SCRF method. ${ }^{c}$ Activation free energy obtained by the SCRF method. ${ }^{\mathrm{d}}$ Activation free energy obtained by the LD method. ${ }^{\mathrm{e}}$ Activation free energy obtained by the AM1-SM1 and PM3-SM3 methods. ${ }^{f}$ The imaginary frequency corresponding to the transition state. ${ }^{g}$ The lowest vibrational frequency corresponding to the reactant state. ${ }^{\mathrm{h}}$ The distance between the reacting N3 atom of methythymine and the BPL carbonyl carbon atom in the transition state structure. ${ }^{\mathrm{i}}$ The distance between the reacting N3 atom of methythymine and the BPL carbonyl carbon atom in the reactant state structure. 
Table S8. The calculated activation parameters for the glutathione alkylation with BPL in the gas phase and solvated with the SCRF and LD models, lowest vibrational frequencies of reactant states, imaginary vibrational frequencies of transition states, and corresponding distances between the reactive centers.

\begin{tabular}{|c|c|c|c|c|c|c|c|c|}
\hline Method/Basis set & $\begin{array}{c}\Delta \boldsymbol{G}_{\text {gas }}^{\ddagger} \\
{[\mathrm{kcal} / \mathrm{mol}]^{\mathrm{a}}}\end{array}$ & $\begin{array}{c}\Delta \Delta G_{h y d r}^{\text {SCRF }} \\
{\left[\mathrm{kcal} / \mathrm{mol}^{\mathrm{b}}{ }^{\mathrm{s}}\right.}\end{array}$ & $\begin{array}{c}\Delta G_{S C R F}^{\ddagger} \\
{[\mathrm{kcal} / \mathrm{mol}]^{\mathrm{c}}}\end{array}$ & $\begin{array}{c}\Delta G_{L D}^{\ddagger} \\
{[\mathrm{kcal} / \mathrm{mol}]^{\mathrm{d}}}\end{array}$ & $\begin{array}{l}\omega^{\mathrm{TS}} \\
{\left[\mathrm{i} \mathbf{~ c m}^{-1}\right]^{\mathrm{e}}}\end{array}$ & $\begin{array}{l}\omega^{\mathrm{R}} \\
{\left[\mathrm{cm}^{-1}\right]^{\mathrm{f}}}\end{array}$ & $\begin{array}{l}d^{\mathrm{IS}} \\
{[\AA]^{\mathrm{g}}}\end{array}$ & $\begin{array}{l}d^{R} \\
{\left[\AA^{R}\right]^{h}}\end{array}$ \\
\hline $\mathrm{HF} / 6-31 \mathrm{G}(\mathrm{d})$ & 22,55 & $\overline{-0,35}$ & 22,20 & 22,78 & 619 & 12 & 2,55 & 3,93 \\
\hline $\mathrm{HF} / 6-31+\mathrm{G}(\mathrm{d}, \mathrm{p})$ & 20,37 & $-1,44$ & 18,93 & 19,51 & 617 & 10 & 2,58 & 4,10 \\
\hline $\mathrm{HF} / 6-311++\mathrm{G}(\mathrm{d}, \mathrm{p})$ & 20,85 & $-1,61$ & 19,24 & 18,78 & 629 & 10 & 2,58 & 4,08 \\
\hline B3LYP/6-31 G(d) & 15,95 & 1,31 & 17,26 & 18,78 & 435 & 13 & 2,61 & 3,73 \\
\hline B3LYP/6-31+ G(d,p) & 1,98 & 3,18 & 5,16 & 5,20 & 443 & 12 & 2,63 & 3,83 \\
\hline B3LYP/6-311++ G(d,p) & 1,78 & 3,21 & 4,99 & 5,28 & 443 & 12 & 2,64 & 3,81 \\
\hline MP2/6-31 G(d) & 23,16 & 0,18 & 23,33 & 25,80 & 583 & 13 & 2,55 & 3,64 \\
\hline MP2/6-31+ G(d,p) & 22,55 & $-3,64$ & 18,91 & 19,75 & 585 & 10 & 2,47 & 3,93 \\
\hline MP2/6-311++G(d,p)* & 22,00 & 2,01 & 24,01 & 22,84 & 585 & 10 & 2,47 & 3,93 \\
\hline M06-2X/6-31 G(d) & 23,57 & 2,15 & 25,72 & 27,63 & 549 & 16 & 2,46 & 3,81 \\
\hline M06-2X/6-31+ G(d,p) & 20,62 & 1,00 & 21,62 & 30,00 & 564 & 14 & 2,48 & 3,70 \\
\hline M06-2X/6-311++ G(d,p) & 20,37 & 1,37 & 21,74 & 22,79 & 571 & 16 & 2,48 & 3,68 \\
\hline mPWPW91/6-31 G(d) & 20,44 & 0,81 & 21,25 & 22,54 & 552 & 13 & 2,49 & 3,68 \\
\hline mPWPW91/6-31+G(d,p) & 18,16 & $-0,32$ & 17,84 & 18,51 & 561 & 13 & 2,51 & 3,74 \\
\hline mPWPW91/6-311++ G(d,p) & 18,38 & 0,44 & 17,94 & 19,80 & 566 & 12 & 2,51 & 3,72 \\
\hline
\end{tabular}

a Gas-phase activation energy. ${ }^{b}$ Hydration free energy of the transition state minus hydration free energy of the reactant state obtained by the SCRF method. ${ }^{c}$ Activation free energy obtained by the SCRF method. ${ }^{\mathrm{d}}$ Activation free energy obtained by the LD method. ${ }^{\mathrm{e}}$ The imaginary frequency corresponding to the transition state. ${ }^{f}$ The lowest vibrational frequency corresponding to the reactant state. ${ }^{\mathrm{g}}$ The distance between the reacting sulfur atom of deprotonated glutathione and BPL $\beta$ carbon atom in the transition state structure. ${ }^{\mathrm{h}}$ The distance between the reacting sulfur atom of deprotonated glutathione and the BPL $\beta$ carbon atom in the reactant state structure. * Activation free energy of alkylation obtained by the SCRF method with single point calculation from MP2 $6-31+G(d, p)$ structure. 
Table S9. The calculated activation parameters for the glutathione acylation with BPL in the gas phase and solvated with the SCRF and LD models, lowest vibrational frequencies of reactant states, imaginary vibrational frequencies of transition states, and corresponding distances between the reactive centers.

\begin{tabular}{|c|c|c|c|c|c|c|c|c|}
\hline Method/Basis set & $\begin{array}{c}\Delta G_{\text {gas }}^{\ddagger} \\
{[\mathrm{kcal} / \mathrm{mol}]^{\mathrm{a}}}\end{array}$ & $\begin{array}{c}\Delta \Delta G_{\text {hydr }}^{\text {SCRF }} \\
{[\mathrm{kcal} / \mathrm{mol}]^{b}}\end{array}$ & $\begin{array}{c}\Delta G_{S C R F}^{\ddagger} \\
{[\mathrm{kcal} / \mathrm{mol}]^{\mathrm{c}}}\end{array}$ & $\begin{array}{c}\Delta G_{L D}^{\ddagger} \\
{[\mathrm{kcal} / \mathrm{mol}]^{\mathrm{d}}}\end{array}$ & $\begin{array}{l}\omega^{\mathrm{TS}} \\
{\left[\mathrm{i} \mathbf{~ c m}^{-1}\right]^{\mathrm{e}}}\end{array}$ & $\begin{array}{l}\omega^{\mathrm{R}} \\
{\left[\mathrm{cm}^{-1}\right]^{\mathrm{f}}}\end{array}$ & $\begin{array}{l}d^{\mathrm{TS}} \\
{[\AA]^{\mathrm{g}}}\end{array}$ & $\begin{array}{l}d^{R} \\
{\left[\AA{ }^{R}\right]^{h}}\end{array}$ \\
\hline $\mathrm{HF} / 6-31 \mathrm{G}(\mathrm{d})$ & 37,44 & $-4,56$ & 32,88 & 29,86 & 387 & 12 & 1,89 & 5,02 \\
\hline $\mathrm{HF} / 6-31+\mathrm{G}(\mathrm{d}, \mathrm{p})$ & 35,21 & $-5,35$ & 29,86 & 27,07 & 410 & 10 & 1,88 & 5,11 \\
\hline $\mathrm{HF} / 6-311++\mathrm{G}(\mathrm{d}, \mathrm{p})$ & 35,14 & $-5,23$ & 29,91 & 25,90 & 414 & 10 & 1,89 & 5,10 \\
\hline B3LYP/6-31 G(d) & 23,07 & $-4,27$ & 18,80 & 16,11 & 166 & 13 & 1,54 & 4,84 \\
\hline B3LYP/6-31+ G(d,p) & 8,54 & $-0,98$ & 7,56 & 7,00 & 173 & 12 & 1,95 & 4,88 \\
\hline B3LYP/6-311++ G(d,p) & 8,53 & $-0,98$ & 7,55 & 7,20 & 148 & 12 & 1,98 & 4,87 \\
\hline MP2/6-31 G(d) & 36,30 & $-22,33$ & 13,97 & 31,66 & 240 & 13 & 1,89 & 4,71 \\
\hline MP2/6-31+ G(d,p) & 35,37 & $-27,71$ & 7,66 & 24,96 & 224 & 10 & 1,90 & 4,91 \\
\hline MP2/6-311++G(d,p) & 35,16 & $-25,34$ & 9,82 & 24,91 & 224 & 10 & 1,90 & 4,91 \\
\hline M06-2X/6-31 G(d) & 25,97 & $-4,99$ & 20,98 & 19,81 & 246 & 16 & 1,89 & 4,74 \\
\hline M06-2X/6-31+ G(d,p) & 22,99 & $-6,66$ & 16,33 & 22,99 & 237 & 14 & 1,89 & 4,68 \\
\hline M06-2X/6-311++ G(d,p) & 22,92 & $-6,56$ & 16,36 & 15,26 & 239 & 16 & 1,90 & 4,66 \\
\hline mPWPW91/6-31 G(d) & 25,13 & $-4,93$ & 20,20 & 17,35 & 233 & 13 & 1,87 & 4,77 \\
\hline mPWPW91/6-31+G(d,p) & 9,72 & $-1,14$ & 8,58 & 7,86 & 263 & 13 & 1,87 & 4,78 \\
\hline mPWPW91/6-311++ G(d,p) & 9,91 & $-1,05$ & 8,86 & 8,05 & 261 & 12 & 1,88 & 4,76 \\
\hline
\end{tabular}

\footnotetext{
${ }^{a}$ Gas-phase activation energy. ${ }^{b}$ Hydration free energy of the transition state minus hydration free energy of the reactant state obtained by the SCRF method. ${ }^{\mathrm{c}}$ Activation free energy obtained by the SCRF method. ${ }^{\mathrm{d}}$ Activation free energy obtained by the LD method. ${ }^{\mathrm{e}}$ The imaginary frequency corresponding to the transition state. ${ }^{\mathrm{f}}$ The lowest vibrational frequency corresponding to the reactant state. ${ }^{\mathrm{g}}$ The distance between the reacting sulfur atom of deprotonated glutathione and BPL carbonyl carbon atom, in the transition state structure. ${ }^{\mathrm{h}}$ The distance between the reacting sulfur atom of deprotonated glutathione and the BPL carbonyl carbon atom in the reactant state structure. * Activation free energy of alkylation obtained by the SCRF method with single point calculation from MP2 6-31+G(d,p) structure.
} 
Table S10. The comparison of the activation free energies for BPL alkylation of the two thymine tautomeric forms used in our calculations.

\begin{tabular}{|c|c|c|c|c|c|c|}
\hline \multirow{2}{*}{ Method/Basis set } & \multirow{2}{*}{$\begin{array}{c}\begin{array}{c}\text { Thymine } \\
\text { [kcal/mol] }\end{array} \\
\Delta G_{\text {gas }}^{\ddagger}{ }^{a}\end{array}$} & \multicolumn{2}{|c|}{ lactam tautomeric form } & $\begin{array}{l}\text { Thymine } \\
\text { [kcal/mol] }\end{array}$ & \multicolumn{2}{|c|}{ lactim tautomeric form } \\
\hline & & $\Delta G_{S C R F}^{\ddagger}{ }^{b}$ & $\Delta G_{L D}^{\ddagger}{ }^{c}$ & $\Delta G_{\text {gas }}^{\ddagger} a$ & $\Delta \boldsymbol{G}_{S C R F}^{\ddagger}{ }^{b}$ & $\Delta G_{L D}^{\ddagger}{ }^{c}$ \\
\hline AM1 & 66,96 & - & - & 54,39 & - & - \\
\hline PM3 & 94,60 & - & - & 60,79 & - & - \\
\hline $\mathrm{HF} / 6-31 \mathrm{G}(\mathrm{d})$ & 71,71 & 47,52 & 41,51 & 55,70 & 33,14 & 40,03 \\
\hline $\mathrm{HF} / 6-31+\mathrm{G}(\mathrm{d}, \mathrm{p})$ & 66,91 & 41,82 & 34,01 & 52,90 & 29,25 & 32,66 \\
\hline $\mathrm{HF} / 6-311++\mathrm{G}(\mathrm{d}, \mathrm{p})$ & 67,16 & 42,61 & 34,25 & 52,93 & 29,75 & 32,95 \\
\hline B3LYP/6-31 G(d) & 60,38 & 39,71 & 31,57 & 46,70 & 27,37 & 30,46 \\
\hline B3LYP/6-31+ G(d,p) & 55,01 & 33,17 & 22,46 & 42,82 & 22,17 & 21,54 \\
\hline B3LYP/6-311++ G(d,p) & 54,71 & 33,19 & 22,98 & 42,05 & 21,72 & 22,09 \\
\hline MP2/6-31 G(d) & 72,23 & 42,32 & 40,25 & 56,36 & 30,29 & 39,32 \\
\hline $\mathrm{MP} 2 / 6-31+\mathrm{G}(\mathrm{d}, \mathrm{p})$ & 66,98 & 34,91 & 33,09 & 53,49 & 25,16 & 31,92 \\
\hline $\mathrm{MP} 2 / 6-311++\mathrm{G}(\mathrm{d}, \mathrm{p})$ & 67,61 & 35,84 & 34,69 & 53,83 & 26,68 & 33,47 \\
\hline M06-2X/6-31 G(d) & 64,34 & 43,51 & 37,05 & 51,90 & 32,52 & 35,34 \\
\hline M06-2X/6-31+ G(d,p) & 59,54 & 37,96 & 29,66 & 42,76 & 28,47 & 28,14 \\
\hline M06-2X/6-311++ G(d,p) & 59,58 & 38,35 & 28,62 & 48,25 & 28,34 & 28,62 \\
\hline mPWPW91/6-31 G(d) & 64,52 & 44,77 & 36,33 & 50,41 & 30,85 & 34,56 \\
\hline mPWPW91/6-31+G(d,p) & 59,89 & 39,33 & 29,05 & 47,59 & 27,09 & 27,50 \\
\hline mPWPW91/6-311++ G(d,p) & 59,76 & 39,61 & 27,97 & 47,06 & 26,97 & 27,97 \\
\hline
\end{tabular}

a Gas-phase activation energy. ${ }^{b}$ Activation free energy obtained by the SCRF-PCM method. ${ }^{c}$ Activation free energy obtained by the LD method. 
Table S11. Absolute energies and Cartesian atomic coordinates for the alkylation reaction of methylguanine at the $\mathrm{M} 06-2 \mathrm{X} / 6-311++\mathrm{G}(\mathrm{d}, \mathrm{p})$ level of theory

Carte sian atomic coordinates $(\AA)$

$E^{\dagger}\left[\right.$ hartree] $\begin{array}{llll}\text { Atom } & \mathbf{x} & \mathbf{y} & \mathbf{z}\end{array}$




\begin{tabular}{lllll}
\hline Transition state $-848,886$ & $\mathrm{~N}$ & -0.302393 & 0.357592 & 0.222139 \\
& $\mathrm{C}$ & -0.302257 & 0.061376 & 1.558216 \\
$\mathrm{C}$ & 0.978968 & 0.321505 & 2.004169 \\
$\mathrm{~N}$ & 1.746122 & 0.766541 & 0.954191 \\
$\mathrm{C}$ & 0.956960 & 0.777742 & -0.090018 \\
$\mathrm{C}$ & 1.305858 & 0.089577 & 3.386429 \\
$\mathrm{~N}$ & 0.148098 & 0.388566 & 4.066099 \\
$\mathrm{C}$ & -1.088574 & 0.601451 & 3.516323 \\
$\mathrm{~N}$ & -1.369818 & 0.384034 & 2.259091 \\
$\mathrm{O}$ & 2.346469 & 0.233947 & 3.972955 \\
$\mathrm{~N}$ & -2.045660 & 1.107351 & 4.341771 \\
$\mathrm{C}$ & -1.446541 & 0.234760 & -0.669256 \\
$\mathrm{H}$ & 0.316369 & 0.629133 & 5.035368 \\
$\mathrm{H}$ & 1.244807 & 1.077491 & -1.086028 \\
$\mathrm{H}$ & -1.977746 & 0.955302 & 5.335077 \\
$\mathrm{H}$ & -2.976716 & 1.120552 & 3.955036 \\
$\mathrm{H}$ & -1.138271 & 0.529690 & -1.670351 \\
$\mathrm{H}$ & -1.793464 & 0.797703 & -0.679626 \\
$\mathrm{H}$ & -2.251214 & 0.883366 & -0.325296 \\
$\mathrm{H}$ & 3.891834 & 0.287725 & 1.400157 \\
$\mathrm{C}$ & 3.592563 & 1.255251 & 1.029093 \\
$\mathrm{H}$ & 3.420332 & 2.020158 & 1.769757 \\
$\mathrm{H}$ & 3.703531 & 0.798835 & -1.101620 \\
$\mathrm{O}$ & 5.556984 & 1.793135 & 1.017606 \\
$\mathrm{C}$ & 3.835270 & 1.629986 & -0.406667 \\
$\mathrm{C}$ & 5.327427 & 1.994506 & -0.223342 \\
$\mathrm{O}$ & 6.045726 & 2.379394 & -1.121232 \\
$\mathrm{H}$ & 3.247797 & 2.489525 & -0.734831 \\
& & & &
\end{tabular}




\begin{tabular}{|c|c|c|c|c|c|}
\hline \multirow[t]{28}{*}{ Reactant state } & - 848,950 & $\mathrm{N}$ & -0.047740 & -0.009359 & 0.225886 \\
\hline & & $\mathrm{C}$ & -0.202011 & 0.147300 & 1.570976 \\
\hline & & $\mathrm{C}$ & 0.824811 & 0.989188 & 1.965734 \\
\hline & & $\mathrm{N}$ & 1.601175 & 1.345340 & 0.891604 \\
\hline & & $\mathrm{C}$ & 1.057861 & 0.734237 & -0.123482 \\
\hline & & $\mathrm{C}$ & 0.968729 & 1.301414 & 3.358047 \\
\hline & & $\mathrm{N}$ & -0.076535 & 0.678408 & 4.099220 \\
\hline & & $\mathrm{C}$ & -1.063779 & -0.123204 & 3.589505 \\
\hline & & $\mathrm{N}$ & -1.178713 & -0.418181 & 2.327476 \\
\hline & & $\mathrm{O}$ & 1.806819 & 1.959245 & 3.932850 \\
\hline & & $\mathrm{N}$ & -1.934288 & -0.667640 & 4.501848 \\
\hline & & $\mathrm{C}$ & -0.884641 & -0.821552 & -0.638040 \\
\hline & & $\mathrm{H}$ & 0.004020 & 0.794138 & 5.101453 \\
\hline & & $\mathrm{H}$ & 1.424327 & 0.772086 & -1.140174 \\
\hline & & $\mathrm{H}$ & -2.158968 & -0.118838 & 5.317753 \\
\hline & & $\mathrm{H}$ & -2.727539 & -1.119705 & 4.071625 \\
\hline & & $\mathrm{H}$ & -0.499724 & -0.750497 & -1.653814 \\
\hline & & $\mathrm{H}$ & -0.860833 & -1.861050 & -0.311081 \\
\hline & & $\mathrm{H}$ & -1.912669 & -0.460405 & -0.610066 \\
\hline & & $\mathrm{H}$ & 3.873752 & 1.625005 & 2.436135 \\
\hline & & $\mathrm{C}$ & 4.631222 & 1.818347 & 1.679069 \\
\hline & & $\mathrm{H}$ & 5.633016 & 1.868857 & 2.101245 \\
\hline & & $\mathrm{H}$ & 3.313006 & 3.417029 & 0.832271 \\
\hline & & $\mathrm{O}$ & 4.574648 & 0.805966 & 0.626955 \\
\hline & & $\mathrm{C}$ & 4.254651 & 2.902773 & 0.663765 \\
\hline & & $\mathrm{C}$ & 4.140114 & 1.717069 & -0.278106 \\
\hline & & $\mathrm{O}$ & 3.806125 & 1.514904 & -1.401934 \\
\hline & & $\mathrm{H}$ & 5.052244 & 3.592244 & 0.390390 \\
\hline
\end{tabular}


Table S12. Absolute energies and Cartesian atomic coordinates for the acylation reaction of methylguanine at the $M 06-2 X / 6-311++G(d, p)$ level of theory

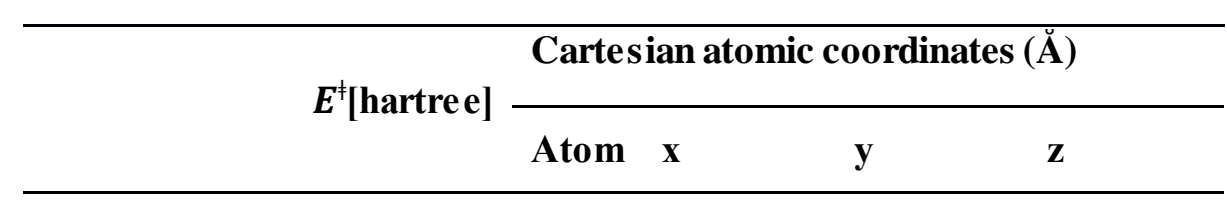




\begin{tabular}{lrrrr}
\hline Transition state 848,875 & N & 0.474625 & -0.064308 & 0.267899 \\
& C & 0.074994 & 0.022131 & 1.581770 \\
C & 1.009468 & 0.803259 & 2.223523 \\
N & 1.950614 & 1.176827 & 1.285348 \\
C & 1.617878 & 0.642074 & 0.125418 \\
C & 0.827169 & 1.146028 & 3.607564 \\
N & -0.333447 & 0.478217 & 4.096170 \\
C & -1.191630 & -0.300391 & 3.367318 \\
N & -1.039388 & -0.546494 & 2.093591 \\
O & 1.469447 & 1.867710 & 4.324108 \\
N & -2.230101 & -0.867300 & 4.042923 \\
C & -0.223208 & -0.805425 & -0.773729 \\
H & -0.494728 & 0.616343 & 5.086207 \\
H & 0.316733 & -0.665699 & -1.707483 \\
H & -0.251774 & -1.862778 & -0.513648 \\
H & -1.239938 & -0.427178 & -0.870069 \\
C & 3.125229 & 3.174810 & -0.342529 \\
H & 4.311058 & 3.710984 & 1.416270 \\
C & 3.319029 & 3.330209 & 1.172607 \\
C & 3.323022 & 1.878726 & 1.577347 \\
O & 4.042025 & 1.260611 & 2.281162 \\
H & 2.562125 & 3.887195 & 1.737649 \\
O & 3.659767 & 1.931147 & -0.598075 \\
H & 3.604404 & 3.996611 & -0.896156 \\
H & 2.036060 & 3.246880 & -0.572690 \\
H & -2.921146 & -1.312653 & 3.459091 \\
H & -2.573270 & -0.430904 & 4.883273 \\
H & 2.303982 & 0.848566 & -0.725209
\end{tabular}




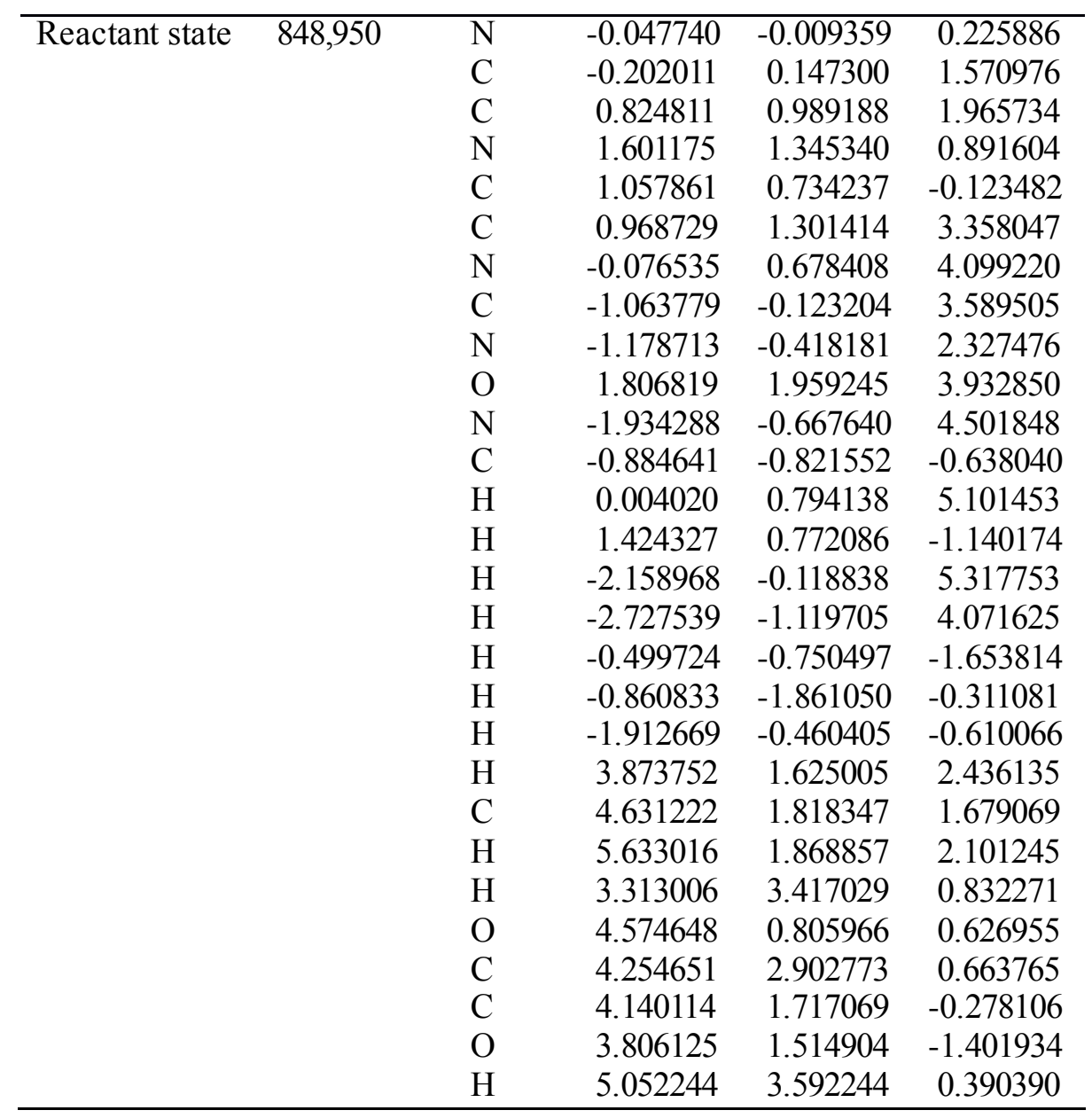


Table S13. Absolute energies and Cartesian atomic coordinates for the alkylation reaction of methyladenine at the M06-2X/6-311++G(d,p) level of theory

\begin{tabular}{|c|c|c|c|c|c|}
\hline & \multirow{2}{*}{$E^{\ddagger}[$ hartree] } & \multicolumn{4}{|c|}{ Cartesian atomic coordinates $(\stackrel{\mathrm{A}}{)})$} \\
\hline & & Atom & $\mathbf{x}$ & $\mathbf{y}$ & $\mathbf{z}$ \\
\hline \multirow[t]{27}{*}{ Transition state } & \multirow{27}{*}{$-773,649$} & $\mathrm{~N}$ & -0.43930 & 0.05153 & 0.28317 \\
\hline & & $\mathrm{C}$ & -0.25016 & 0.01558 & 1.61007 \\
\hline & & $\mathrm{C}$ & 0.95827 & 0.15400 & 2.28275 \\
\hline & & $\mathrm{C}$ & 2.10429 & 0.34599 & 1.49175 \\
\hline & & $\mathrm{N}$ & 1.92095 & 0.39204 & 0.16121 \\
\hline & & $\mathrm{C}$ & 0.68625 & 0.24230 & -0.36539 \\
\hline & & $\mathrm{N}$ & -1.18476 & -0.17305 & 2.58788 \\
\hline & & $\mathrm{C}$ & -0.49679 & -0.13684 & 3.77703 \\
\hline & & $\mathrm{N}$ & 0.78804 & 0.05452 & 3.64127 \\
\hline & & $\mathrm{N}$ & 3.32275 & 0.49001 & 2.03406 \\
\hline & & $\mathrm{H}$ & 0.64202 & 0.28644 & -1.44931 \\
\hline & & $\mathrm{H}$ & -1.01100 & -0.26400 & 4.71922 \\
\hline & & $\mathrm{C}$ & -2.61263 & -0.35111 & 2.37723 \\
\hline & & $\mathrm{H}$ & -2.77205 & -1.11848 & 1.62099 \\
\hline & & $\mathrm{H}$ & -3.06629 & -0.66286 & 3.31640 \\
\hline & & $\mathrm{H}$ & -3.06358 & 0.58302 & 2.04229 \\
\hline & & $\mathrm{H}$ & 4.15530 & 0.52010 & 1.46914 \\
\hline & & $\mathrm{H}$ & 3.41354 & 0.39680 & 3.03375 \\
\hline & & $\mathrm{H}$ & 3.82396 & 1.42489 & -0.45286 \\
\hline & & $\mathrm{H}$ & 4.29377 & -1.12512 & -0.10690 \\
\hline & & $\mathrm{C}$ & 3.94637 & -0.75703 & -1.07417 \\
\hline & & $\mathrm{C}$ & 3.37706 & 0.64188 & -1.04688 \\
\hline & & $\mathrm{C}$ & 5.09527 & -0.41632 & -2.04229 \\
\hline & & $\mathrm{O}$ & 4.91214 & 0.83012 & -2.29734 \\
\hline & & $\mathrm{O}$ & 5.94100 & -1.19020 & -2.42861 \\
\hline & & $\mathrm{H}$ & 3.24729 & -1.48510 & -1.48775 \\
\hline & & $\mathrm{H}$ & 2.72103 & 0.97156 & -1.83825 \\
\hline
\end{tabular}




\begin{tabular}{|c|c|c|c|c|c|}
\hline \multirow[t]{27}{*}{ Reactant state } & \multirow[t]{27}{*}{$-773,711$} & $\mathrm{~N}$ & -1.72447 & -1.16325 & -0.03291 \\
\hline & & $\mathrm{C}$ & -0.43485 & -0.76067 & -0.30204 \\
\hline & & $\mathrm{C}$ & -0.33456 & 0.62526 & -0.31559 \\
\hline & & $\mathrm{N}$ & -1.59876 & 1.08032 & -0.04923 \\
\hline & & $\mathrm{C}$ & -2.37632 & -0.04211 & 0.11036 \\
\hline & & $\mathrm{N}$ & 0.76898 & 1.34736 & -0.53998 \\
\hline & & $\mathrm{C}$ & 1.81611 & 0.56553 & -0.75415 \\
\hline & & $\mathrm{N}$ & 1.87962 & -0.77467 & -0.77032 \\
\hline & & $\mathrm{C}$ & 0.75519 & -1.47334 & -0.54836 \\
\hline & & $\mathrm{N}$ & 0.78243 & -2.81781 & -0.59135 \\
\hline & & $\mathrm{H}$ & 2.75624 & 1.07677 & -0.94479 \\
\hline & & $\mathrm{C}$ & -1.99381 & 2.47208 & 0.05377 \\
\hline & & $\mathrm{H}$ & -3.43075 & 0.04303 & 0.33313 \\
\hline & & $\mathrm{H}$ & 1.68074 & -3.28293 & -0.59894 \\
\hline & & $\mathrm{H}$ & -0.03268 & -3.31776 & -0.27490 \\
\hline & & $\mathrm{H}$ & -3.06969 & 2.51763 & 0.21602 \\
\hline & & $\mathrm{H}$ & -1.47761 & 2.94851 & 0.88756 \\
\hline & & $\mathrm{H}$ & -1.74074 & 2.99752 & -0.86692 \\
\hline & & $\mathrm{H}$ & 5.33245 & -2.95080 & -1.85254 \\
\hline & & $\mathrm{C}$ & 4.52502 & -2.39249 & -1.38337 \\
\hline & & $\mathrm{H}$ & 3.87221 & -1.91260 & -2.10819 \\
\hline & & $\mathrm{H}$ & 5.94016 & -1.56619 & 0.14029 \\
\hline & & $\mathrm{O}$ & 3.74250 & -3.27075 & -0.51725 \\
\hline & & $\mathrm{C}$ & 4.89957 & -1.52384 & -0.17752 \\
\hline & & $\mathrm{C}$ & 3.99804 & -2.48488 & 0.57664 \\
\hline & & $\mathrm{O}$ & 3.58144 & -2.63583 & 1.67287 \\
\hline & & $\mathrm{H}$ & 4.52703 & -0.50312 & -0.18184 \\
\hline
\end{tabular}


Table S14. Absolute energies and Cartesian atomic coordinates for the acylation reaction of methyladenine at the $M 06-2 X / 6-311++G(d, p)$ level of theory

\begin{tabular}{|c|c|c|c|c|c|}
\hline & \multirow{2}{*}{$E^{\ddagger}[$ hartree $]$} & \multicolumn{4}{|c|}{ Cartesian atomic coordinates $(\stackrel{\mathrm{A}}{)})$} \\
\hline & & Atom & $\mathbf{x}$ & $\mathbf{y}$ & $\mathbf{z}$ \\
\hline \multirow{27}{*}{ Transition state } & $-773,660$ & $\mathrm{C}$ & -1.645869 & -1.219486 & 1.108091 \\
\hline & & $\mathrm{N}$ & -1.111765 & -0.636966 & 2.205350 \\
\hline & & $\mathrm{C}$ & -0.099181 & 0.123085 & 1.903724 \\
\hline & & $\mathrm{N}$ & 0.405495 & 0.376814 & 0.663478 \\
\hline & & $\mathrm{C}$ & -0.115611 & -0.221969 & -0.466865 \\
\hline & & $\mathrm{C}$ & -1.226101 & -1.071309 & -0.199345 \\
\hline & & $\mathrm{N}$ & 0.399088 & 0.006317 & -1.642732 \\
\hline & & $\mathrm{C}$ & 1.276740 & 2.856346 & -0.676036 \\
\hline & & $\mathrm{H}$ & 2.267789 & 3.284741 & 1.231912 \\
\hline & & $\mathrm{C}$ & 1.404115 & 2.747925 & 0.840145 \\
\hline & & $\mathrm{C}$ & 1.784458 & 1.264543 & 0.770310 \\
\hline & & $\mathrm{O}$ & 2.671191 & 0.687062 & 1.318598 \\
\hline & & $\mathrm{H}$ & 0.519143 & 2.966886 & 1.439452 \\
\hline & & $\mathrm{O}$ & 1.994151 & 1.667024 & -0.981330 \\
\hline & & $\mathrm{H}$ & 1.741545 & 3.735870 & -1.123295 \\
\hline & & $\mathrm{H}$ & 0.233989 & 2.790592 & -1.014591 \\
\hline & & $\mathrm{H}$ & -0.047204 & -0.472291 & -2.412701 \\
\hline & & $\mathrm{H}$ & 1.263584 & 0.818820 & -1.577837 \\
\hline & & $\mathrm{H}$ & -2.914280 & -2.365843 & 3.094517 \\
\hline & & $\mathrm{C}$ & -3.493765 & -2.536167 & 2.188732 \\
\hline & & $\mathrm{N}$ & -2.697218 & -2.089372 & 1.057771 \\
\hline & & $\mathrm{H}$ & -4.426696 & -1.974531 & 2.247633 \\
\hline & & $\mathrm{C}$ & -2.853339 & -2.411620 & -0.269088 \\
\hline & & $\mathrm{H}$ & -3.707711 & -3.599277 & 2.086586 \\
\hline & & $\mathrm{N}$ & -1.990251 & -1.823577 & -1.054307 \\
\hline & & $\mathrm{H}$ & -3.627471 & -3.093854 & -0.590047 \\
\hline & & $\mathrm{H}$ & 0.430863 & 0.619976 & 2.709394 \\
\hline \multirow[t]{2}{*}{ Reactant state } & $-773,711$ & $\mathrm{~N}$ & -1.72447 & -1.16325 & -0.03291 \\
\hline & & $\mathrm{C}$ & -0.43485 & -0.76067 & -0.30204 \\
\hline
\end{tabular}




\begin{tabular}{rrrr}
\hline C & -0.33456 & 0.62526 & -0.31559 \\
N & -1.59876 & 1.08032 & -0.04923 \\
C & -2.37632 & -0.04211 & 0.11036 \\
N & 0.76898 & 1.34736 & -0.53998 \\
C & 1.81611 & 0.56553 & -0.75415 \\
N & 1.87962 & -0.77467 & -0.77032 \\
C & 0.75519 & -1.47334 & -0.54836 \\
N & 0.78243 & -2.81781 & -0.59135 \\
H & 2.75624 & 1.07677 & -0.94479 \\
C & -1.99381 & 2.47208 & 0.05377 \\
H & -3.43075 & 0.04303 & 0.33313 \\
H & 1.68074 & -3.28293 & -0.59894 \\
H & -0.03268 & -3.31776 & -0.27490 \\
H & -3.06969 & 2.51763 & 0.21602 \\
H & -1.47761 & 2.94851 & 0.88756 \\
H & -1.74074 & 2.99752 & -0.86692 \\
H & 5.33245 & -2.95080 & -1.85254 \\
C & 4.52502 & -2.39249 & -1.38337 \\
H & 3.87221 & -1.91260 & -2.10819 \\
H & 5.94016 & -1.56619 & 0.14029 \\
O & 3.74250 & -3.27075 & -0.51725 \\
C & 4.89957 & -1.52384 & -0.17752 \\
C & 3.99804 & -2.48488 & 0.57664 \\
O & 3.58144 & -2.63583 & 1.67287 \\
H & 4.52703 & -0.50312 & -0.18184 \\
\hline & & & \\
\hline
\end{tabular}


Table S15. Absolute energies and Cartesian atomic coordinates for the alkylation reaction of methylcytosine at the M06-2X/6-311++G(d,p) level of theory

\begin{tabular}{|c|c|c|c|c|}
\hline \multirow{2}{*}{$E^{\dagger}[$ hartree] } & \multicolumn{4}{|c|}{ Cartesian atomic coordinates $(\stackrel{\mathrm{A}}{)})$} \\
\hline & Atom & $\mathbf{x}$ & $\mathbf{y}$ & $\mathbf{z}$ \\
\hline
\end{tabular}




\begin{tabular}{|c|c|c|c|c|c|}
\hline \multirow[t]{25}{*}{ Transition state } & \multirow[t]{25}{*}{$-701,272$} & $\mathrm{~N}$ & -0.12342 & -0.55336 & 0.46008 \\
\hline & & $\mathrm{C}$ & -0.63189 & 0.55706 & -0.05271 \\
\hline & & $\mathrm{C}$ & 0.20662 & 1.56142 & -0.62681 \\
\hline & & $\mathrm{C}$ & 1.54109 & 1.32939 & -0.60406 \\
\hline & & $\mathrm{N}$ & 2.06123 & 0.20563 & -0.06652 \\
\hline & & $\mathrm{C}$ & 1.23549 & -0.78673 & 0.50306 \\
\hline & & $\mathrm{N}$ & -1.96425 & 0.73116 & -0.03850 \\
\hline & & $\mathrm{O}$ & 1.73029 & -1.77361 & 0.99018 \\
\hline & & $\mathrm{H}$ & -0.20238 & 2.46491 & -1.05247 \\
\hline & & $\mathrm{H}$ & 2.25354 & 2.03600 & -1.01249 \\
\hline & & $\mathrm{H}$ & -2.56705 & 0.08571 & 0.44653 \\
\hline & & $\mathrm{H}$ & -2.36491 & 1.58931 & -0.37633 \\
\hline & & $\mathrm{C}$ & 3.50045 & -0.04938 & -0.02135 \\
\hline & & $\mathrm{H}$ & 3.82531 & -0.13575 & 1.01496 \\
\hline & & $\mathrm{H}$ & 3.72046 & -0.98644 & -0.53100 \\
\hline & & $\mathrm{H}$ & 4.01487 & 0.77541 & -0.51045 \\
\hline & & $\mathrm{H}$ & -0.45257 & -2.69089 & 1.04799 \\
\hline & & $\mathrm{H}$ & -0.63577 & -1.36187 & 3.15452 \\
\hline & & $\mathrm{C}$ & -1.52845 & -1.39401 & 2.52873 \\
\hline & & $\mathrm{C}$ & -1.22908 & -1.95097 & 1.15827 \\
\hline & & $\mathrm{C}$ & -2.49491 & -2.52301 & 2.93730 \\
\hline & & $\mathrm{O}$ & -2.47232 & -3.32731 & 1.93840 \\
\hline & & $\mathrm{O}$ & -3.11219 & -2.56465 & 3.97915 \\
\hline & & $\mathrm{H}$ & -1.99513 & -0.40658 & 2.53714 \\
\hline & & $\mathrm{H}$ & -1.93759 & -1.87572 & 0.34658 \\
\hline \multirow[t]{7}{*}{ Reactant state } & \multirow[t]{7}{*}{$-701,339$} & $\mathrm{~N}$ & -0.41778 & -0.50081 & 1.12897 \\
\hline & & $\mathrm{C}$ & -1.04139 & 0.50051 & 0.53765 \\
\hline & & $\mathrm{C}$ & -0.37105 & 1.41463 & -0.34629 \\
\hline & & $\mathrm{C}$ & 0.95053 & 1.20344 & -0.53932 \\
\hline & & $\mathrm{N}$ & 1.59995 & 0.18161 & 0.06621 \\
\hline & & $\mathrm{C}$ & 0.90936 & -0.71339 & 0.92191 \\
\hline & & $\mathrm{N}$ & -2.35309 & 0.65968 & 0.78259 \\
\hline
\end{tabular}




\begin{tabular}{rrrr}
\hline $\mathrm{O}$ & 1.54063 & -1.62868 & 1.42514 \\
$\mathrm{H}$ & -0.88737 & 2.23051 & -0.82988 \\
$\mathrm{H}$ & 1.55138 & 1.83637 & -1.18190 \\
$\mathrm{H}$ & -2.82053 & 0.02956 & 1.42654 \\
$\mathrm{H}$ & -2.86763 & 1.41139 & 0.36010 \\
$\mathrm{C}$ & 3.02226 & -0.06353 & -0.13644 \\
$\mathrm{H}$ & 3.54401 & -0.02413 & 0.81945 \\
$\mathrm{H}$ & 3.17000 & -1.05523 & -0.56374 \\
$\mathrm{H}$ & 3.41669 & 0.69599 & -0.80981 \\
$\mathrm{H}$ & -1.20523 & -4.71951 & 1.65950 \\
$\mathrm{H}$ & -1.28064 & -3.56693 & 3.88916 \\
$\mathrm{C}$ & -1.07706 & -2.93957 & 3.02283 \\
$\mathrm{C}$ & -1.05272 & -3.64214 & 1.66232 \\
$\mathrm{C}$ & -2.30829 & -2.27280 & 2.44667 \\
$\mathrm{O}$ & -2.28964 & -2.95779 & 1.28282 \\
$\mathrm{O}$ & -3.09497 & -1.43455 & 2.76868 \\
$\mathrm{H}$ & -0.25687 & -2.24751 & 3.19919 \\
$\mathrm{H}$ & -0.23493 & -3.33391 & 1.01672 \\
\hline
\end{tabular}


Table S16. Absolute energies and Cartesian atomic coordinates for the acylation reaction of methylcytosine at the M06-2X/6-311++G(d,p) level of theory

\begin{tabular}{|c|c|c|c|c|c|}
\hline & \multirow{2}{*}{$E^{\dagger}[$ hartree] } & \multicolumn{4}{|c|}{ Cartesian atomic coordinates $(\AA)$} \\
\hline & & Atom & $\mathbf{x}$ & $\mathbf{y}$ & $\mathbf{z}$ \\
\hline \multirow[t]{24}{*}{ Transition state } & \multirow[t]{24}{*}{$-701,285$} & $\mathrm{C}$ & -1.620635 & -1.361064 & 1.160391 \\
\hline & & $\mathrm{N}$ & -1.010031 & -0.725932 & 2.195127 \\
\hline & & $\mathrm{C}$ & 0.038852 & 0.184483 & 1.976247 \\
\hline & & $\mathrm{N}$ & 0.406634 & 0.408706 & 0.666724 \\
\hline & & $\mathrm{C}$ & -0.205046 & -0.199176 & -0.388626 \\
\hline & & $\mathrm{C}$ & -1.267527 & -1.138526 & -0.122512 \\
\hline & & $\mathrm{O}$ & 0.563159 & 0.732465 & 2.926161 \\
\hline & & $\mathrm{N}$ & 0.185290 & 0.115231 & -1.597345 \\
\hline & & $\mathrm{C}$ & 1.355522 & 2.871293 & -0.538009 \\
\hline & & $\mathrm{H}$ & 2.778056 & 3.032431 & 1.117696 \\
\hline & & $\mathrm{C}$ & 1.797262 & 2.603906 & 0.896592 \\
\hline & & $\mathrm{C}$ & 2.009914 & 1.122201 & 0.613404 \\
\hline & & $\mathrm{O}$ & 2.847876 & 0.334140 & 0.890086 \\
\hline & & $\mathrm{H}$ & 1.103939 & 2.803326 & 1.713386 \\
\hline & & $\mathrm{O}$ & 1.930771 & 1.696618 & -1.095727 \\
\hline & & $\mathrm{H}$ & 1.759257 & 3.778233 & -0.996939 \\
\hline & & $\mathrm{H}$ & 0.260228 & 2.874565 & -0.642660 \\
\hline & & $\mathrm{H}$ & -0.261472 & -0.364534 & -2.366452 \\
\hline & & $\mathrm{H}$ & 1.070138 & 0.925095 & -1.594264 \\
\hline & & $\mathrm{C}$ & -1.383068 & -0.950612 & 3.586382 \\
\hline & & $\mathrm{H}$ & -1.765299 & -1.641784 & -0.940362 \\
\hline & & $\mathrm{H}$ & -2.411415 & -2.052668 & 1.432972 \\
\hline & & $\mathrm{H}$ & -2.188266 & -1.685557 & 3.616034 \\
\hline & & $\mathrm{H}$ & -1.714845 & -0.013829 & 4.038084 \\
\hline
\end{tabular}




\begin{tabular}{|c|c|c|c|c|c|}
\hline & & $\mathrm{H}$ & -0.520365 & -1.315561 & 4.146372 \\
\hline \multirow[t]{25}{*}{ Reactant state } & $-701,339$ & $\mathrm{~N}$ & -0.41778 & -0.50081 & 1.12897 \\
\hline & & $\mathrm{C}$ & -1.04139 & 0.50051 & 0.53765 \\
\hline & & $\mathrm{C}$ & -0.37105 & 1.41463 & -0.34629 \\
\hline & & $\mathrm{C}$ & 0.95053 & 1.20344 & -0.53932 \\
\hline & & $\mathrm{N}$ & 1.59995 & 0.18161 & 0.06621 \\
\hline & & $\mathrm{C}$ & 0.90936 & -0.71339 & 0.92191 \\
\hline & & $\mathrm{N}$ & -2.35309 & 0.65968 & 0.78259 \\
\hline & & $\mathrm{O}$ & 1.54063 & -1.62868 & 1.42514 \\
\hline & & $\mathrm{H}$ & -0.88737 & 2.23051 & -0.82988 \\
\hline & & $\mathrm{H}$ & 1.55138 & 1.83637 & -1.18190 \\
\hline & & $\mathrm{H}$ & -2.82053 & 0.02956 & 1.42654 \\
\hline & & $\mathrm{H}$ & -2.86763 & 1.41139 & 0.36010 \\
\hline & & $\mathrm{C}$ & 3.02226 & -0.06353 & -0.13644 \\
\hline & & $\mathrm{H}$ & 3.54401 & -0.02413 & 0.81945 \\
\hline & & $\mathrm{H}$ & 3.17000 & -1.05523 & -0.56374 \\
\hline & & $\mathrm{H}$ & 3.41669 & 0.69599 & -0.80981 \\
\hline & & $\mathrm{H}$ & -1.20523 & -4.71951 & 1.65950 \\
\hline & & $\mathrm{H}$ & -1.28064 & -3.56693 & 3.88916 \\
\hline & & $\mathrm{C}$ & -1.07706 & -2.93957 & 3.02283 \\
\hline & & $\mathrm{C}$ & -1.05272 & -3.64214 & 1.66232 \\
\hline & & $\mathrm{C}$ & -2.30829 & -2.27280 & 2.44667 \\
\hline & & $\mathrm{O}$ & -2.28964 & -2.95779 & 1.28282 \\
\hline & & $\mathrm{O}$ & -3.09497 & -1.43455 & 2.76868 \\
\hline & & $\mathrm{H}$ & -0.25687 & -2.24751 & 3.19919 \\
\hline & & $\mathrm{H}$ & -0.23493 & -3.33391 & 1.01672 \\
\hline
\end{tabular}


Table S17. Absolute energies and Cartesian atomic coordinates for the alkylation reaction of methylthymine lactim tautomeric form at the M06$2 \mathrm{X} / 6-311++\mathrm{G}(\mathrm{d}, \mathrm{p})$ level of theory

\begin{tabular}{|c|c|c|c|c|c|}
\hline & \multirow{2}{*}{$E^{\dagger}[$ hartree] } & \multicolumn{4}{|c|}{ Cartesian atomic coordinates (Ă) } \\
\hline & & Atom & $\mathbf{x}$ & $\mathbf{y}$ & $\mathbf{z}$ \\
\hline \multirow[t]{24}{*}{ Transition state } & \multirow[t]{24}{*}{$-760,444$} & $\mathrm{C}$ & 2.228723 & 1.026875 & 0.409938 \\
\hline & & $\mathrm{C}$ & 3.293421 & 0.193349 & -0.033965 \\
\hline & & $\mathrm{C}$ & 3.906770 & 0.624055 & -1.167461 \\
\hline & & $\mathrm{N}$ & 3.536106 & 1.752706 & -1.818315 \\
\hline & & $\mathrm{C}$ & 2.481876 & 2.558358 & -1.364509 \\
\hline & & $\mathrm{N}$ & 1.862812 & 2.130670 & -0.198182 \\
\hline & & $\mathrm{C}$ & 3.665884 & -1.054002 & 0.710709 \\
\hline & & $\mathrm{O}$ & 2.157280 & 3.551277 & -1.966719 \\
\hline & & $\mathrm{O}$ & 1.598092 & 0.629189 & 1.504405 \\
\hline & & $\mathrm{C}$ & 4.198945 & 2.199469 & -3.044541 \\
\hline & & $\mathrm{H}$ & 4.500914 & -1.553119 & 0.218670 \\
\hline & & $\mathrm{H}$ & 2.823841 & -1.747066 & 0.754838 \\
\hline & & $\mathrm{H}$ & 3.954827 & -0.823451 & 1.737690 \\
\hline & & $\mathrm{H}$ & 4.729490 & 0.073771 & -1.609831 \\
\hline & & $\mathrm{H}$ & 0.852241 & 1.208318 & 1.712041 \\
\hline & & $\mathrm{H}$ & 4.617476 & 3.193401 & -2.890591 \\
\hline & & $\mathrm{H}$ & 4.987511 & 1.492100 & -3.291329 \\
\hline & & $\mathrm{H}$ & 3.470866 & 2.252690 & -3.853081 \\
\hline & & $\mathrm{H}$ & 0.738187 & 4.058097 & -0.153877 \\
\hline & & $\mathrm{C}$ & 0.436032 & 3.207083 & 0.435827 \\
\hline & & $\mathrm{H}$ & -0.824069 & 2.336701 & -1.096510 \\
\hline & & $\mathrm{O}$ & -1.151609 & 4.269224 & 1.141844 \\
\hline & & $\mathrm{C}$ & -0.753141 & 2.390526 & -0.009569 \\
\hline & & $\mathrm{H}$ & 0.777040 & 3.202516 & 1.462334 \\
\hline
\end{tabular}




\begin{tabular}{|c|c|c|c|c|c|}
\hline & $\mathrm{C}$ & -1.823442 & 3.317655 & 0.611301 \\
\hline & & $\mathrm{O}$ & -3.015059 & 3.105906 & 0.566894 \\
\hline & & $\mathrm{H}$ & -0.800846 & 1.374802 & 0.391780 \\
\hline \multirow[t]{27}{*}{ Reactant state } & \multirow[t]{27}{*}{$-760,521$} & $\mathrm{C}$ & -1.407287 & -1.318594 & 1.210110 \\
\hline & & $\mathrm{N}$ & -0.768908 & -0.677544 & 2.225979 \\
\hline & & $\mathrm{C}$ & 0.049447 & 0.439502 & 1.983503 \\
\hline & & $\mathrm{N}$ & 0.190985 & 0.839145 & 0.682769 \\
\hline & & $\mathrm{C}$ & -0.431912 & 0.209050 & -0.274455 \\
\hline & & $\mathrm{C}$ & -1.286528 & -0.927076 & -0.080957 \\
\hline & & $\mathrm{O}$ & 0.591501 & 0.995825 & 2.924170 \\
\hline & & $\mathrm{O}$ & -0.289135 & 0.612728 & -1.526141 \\
\hline & & $\mathrm{C}$ & 1.143031 & 3.942519 & 1.597915 \\
\hline & & $\mathrm{H}$ & 3.265075 & 3.711469 & 0.908309 \\
\hline & & $\mathrm{C}$ & 2.325298 & 3.170417 & 1.003374 \\
\hline & & $\mathrm{C}$ & 1.469082 & 3.190435 & -0.243615 \\
\hline & & $\mathrm{O}$ & 1.515641 & 2.749746 & -1.355270 \\
\hline & & $\mathrm{H}$ & 2.467894 & 2.167683 & 1.400428 \\
\hline & & $\mathrm{O}$ & 0.473897 & 3.918469 & 0.293949 \\
\hline & & $\mathrm{H}$ & 1.328261 & 4.970158 & 1.903281 \\
\hline & & $\mathrm{H}$ & 0.565145 & 3.382288 & 2.328345 \\
\hline & & $\mathrm{C}$ & -0.912255 & -1.097713 & 3.615097 \\
\hline & & $\mathrm{H}$ & 0.070741 & -1.298541 & 4.039813 \\
\hline & & $\mathrm{H}$ & -1.526669 & -1.995753 & 3.648738 \\
\hline & & $\mathrm{H}$ & -1.378421 & -0.302452 & 4.197220 \\
\hline & & $\mathrm{H}$ & 0.311124 & 1.385912 & -1.563087 \\
\hline & & $\mathrm{C}$ & -1.965014 & -1.596610 & -1.237451 \\
\hline & & $\mathrm{H}$ & -1.231513 & -1.964980 & -1.957529 \\
\hline & & $\mathrm{H}$ & -2.611693 & -0.894540 & -1.767548 \\
\hline & & $\mathrm{H}$ & -2.569604 & -2.437218 & -0.894089 \\
\hline & & $\mathrm{H}$ & -2.020422 & -2.166066 & 1.495979 \\
\hline
\end{tabular}


Table S18. Absolute energies and Cartesian atomic coordinates for the acylation reaction of methylthymine lactim tautomeric form at the M06$2 \mathrm{X} / 6-311++\mathrm{G}(\mathrm{d}, \mathrm{p})$ level of theory

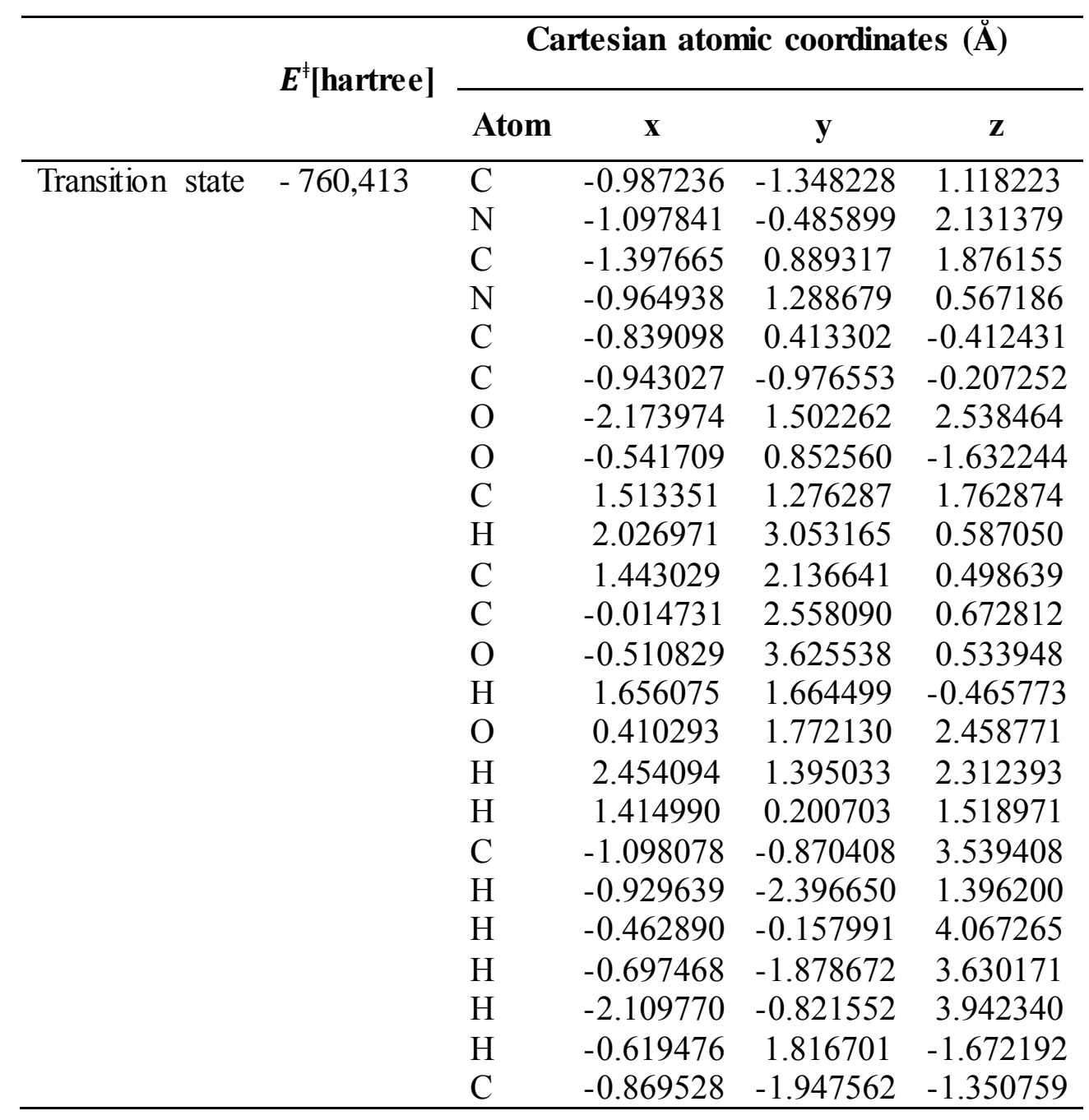




\begin{tabular}{|c|c|c|c|c|c|}
\hline & $\mathrm{H}$ & 0.053541 & -1.822328 & -1.920112 \\
\hline & & $\mathrm{H}$ & -1.704425 & -1.810354 & -2.040880 \\
\hline & & $\mathrm{H}$ & -0.905504 & -2.971431 & -0.976934 \\
\hline \multirow[t]{27}{*}{ Reactant state } & \multirow[t]{27}{*}{$-760,521$} & $\mathrm{C}$ & -1.407287 & -1.318594 & 1.210110 \\
\hline & & $\mathrm{N}$ & -0.768908 & -0.677544 & 2.225979 \\
\hline & & $\mathrm{C}$ & 0.049447 & 0.439502 & 1.983503 \\
\hline & & $\mathrm{N}$ & 0.190985 & 0.839145 & 0.682769 \\
\hline & & $\mathrm{C}$ & -0.431912 & 0.209050 & -0.274455 \\
\hline & & $\mathrm{C}$ & -1.286528 & -0.927076 & -0.080957 \\
\hline & & $\mathrm{O}$ & 0.591501 & 0.995825 & 2.924170 \\
\hline & & $\mathrm{O}$ & -0.289135 & 0.612728 & -1.526141 \\
\hline & & $\mathrm{C}$ & 1.143031 & 3.942519 & 1.597915 \\
\hline & & $\mathrm{H}$ & 3.265075 & 3.711469 & 0.908309 \\
\hline & & $\mathrm{C}$ & 2.325298 & 3.170417 & 1.003374 \\
\hline & & $\mathrm{C}$ & 1.469082 & 3.190435 & -0.243615 \\
\hline & & $\mathrm{O}$ & 1.515641 & 2.749746 & -1.355270 \\
\hline & & $\mathrm{H}$ & 2.467894 & 2.167683 & 1.400428 \\
\hline & & $\mathrm{O}$ & 0.473897 & 3.918469 & 0.293949 \\
\hline & & $\mathrm{H}$ & 1.328261 & 4.970158 & 1.903281 \\
\hline & & $\mathrm{H}$ & 0.565145 & 3.382288 & 2.328345 \\
\hline & & $\mathrm{C}$ & -0.912255 & -1.097713 & 3.615097 \\
\hline & & $\mathrm{H}$ & 0.070741 & -1.298541 & 4.039813 \\
\hline & & $\mathrm{H}$ & -1.526669 & -1.995753 & 3.648738 \\
\hline & & $\mathrm{H}$ & -1.378421 & -0.302452 & 4.197220 \\
\hline & & $\mathrm{H}$ & 0.311124 & 1.385912 & -1.563087 \\
\hline & & $\mathrm{C}$ & -1.965014 & -1.596610 & -1.237451 \\
\hline & & $\mathrm{H}$ & -1.231513 & -1.964980 & -1.957529 \\
\hline & & $\mathrm{H}$ & -2.611693 & -0.894540 & -1.767548 \\
\hline & & $\mathrm{H}$ & -2.569604 & -2.437218 & -0.894089 \\
\hline & & $\mathrm{H}$ & -2.020422 & -2.166066 & 1.495979 \\
\hline
\end{tabular}


Table S19. Absolute energies and Cartesian atomic coordinates for the alkyation reaction of glutathione at the $M 06-2 X / 6-311++G(d, p)$ level of theory

\begin{tabular}{|c|c|c|c|c|c|}
\hline & \multirow{2}{*}{$E^{\ddagger}[$ hartree] } & \multicolumn{4}{|c|}{ Cartesian atomic coordinates $(\stackrel{\mathrm{A}}{)})$} \\
\hline & & Atom & $\mathbf{x}$ & $\mathbf{y}$ & $\mathbf{z}$ \\
\hline \multirow[t]{24}{*}{ Transition state } & \multirow[t]{24}{*}{-1671.076} & $\mathrm{~S}$ & -1.456648 & -3.121415 & -2.279412 \\
\hline & & $\mathrm{O}$ & 0.017973 & 1.951763 & -2.159099 \\
\hline & & $\mathrm{O}$ & -3.441805 & 0.350286 & -0.828665 \\
\hline & & $\mathrm{O}$ & 4.704819 & 1.138147 & -0.164961 \\
\hline & & $\mathrm{O}$ & 4.833606 & 0.705814 & 2.051969 \\
\hline & & $\mathrm{O}$ & 0.153599 & -1.111577 & 2.141200 \\
\hline & & $\mathrm{O}$ & -0.615678 & 0.665686 & 3.256476 \\
\hline & & $\mathrm{N}$ & -0.206020 & -0.303056 & -2.221305 \\
\hline & & $\mathrm{N}$ & 2.298139 & 0.251267 & 2.341261 \\
\hline & & $\mathrm{N}$ & -1.579454 & -0.494108 & 0.156564 \\
\hline & & $\mathrm{C}$ & 1.815369 & 1.016021 & 0.050854 \\
\hline & & $\mathrm{C}$ & 1.896436 & 0.647527 & -1.444213 \\
\hline & & $\mathrm{C}$ & -1.654280 & -0.309842 & -2.288808 \\
\hline & & $\mathrm{C}$ & 2.794299 & 0.254359 & 0.933753 \\
\hline & & $\mathrm{C}$ & 0.501547 & 0.840744 & -2.008340 \\
\hline & & $\mathrm{C}$ & -2.149020 & -1.584517 & -2.986818 \\
\hline & & $\mathrm{C}$ & -2.319500 & -0.128876 & -0.908470 \\
\hline & & $\mathrm{C}$ & 4.271722 & 0.767150 & 0.932191 \\
\hline & & $\mathrm{C}$ & -1.986948 & -0.288680 & 1.531205 \\
\hline & & $\mathrm{C}$ & -0.724665 & -0.225269 & 2.407259 \\
\hline & & $\mathrm{H}$ & 0.802729 & 0.793437 & 0.404370 \\
\hline & & $\mathrm{H}$ & 1.967940 & 2.092851 & 0.164126 \\
\hline & & $\mathrm{H}$ & 2.616638 & 1.287739 & -1.950556 \\
\hline & & $\mathrm{H}$ & 2.224971 & -0.388705 & -1.560184 \\
\hline
\end{tabular}




\begin{tabular}{|c|c|c|c|c|c|}
\hline & & $\mathrm{H}$ & -1.972962 & 0.565302 & -2.858895 \\
\hline & & $\mathrm{H}$ & 0.225489 & -1.208663 & -2.073203 \\
\hline & & $\mathrm{H}$ & 3.134277 & 0.094967 & 2.917817 \\
\hline & & $\mathrm{H}$ & 1.455431 & -0.451520 & 2.437272 \\
\hline & & $\mathrm{H}$ & -0.773316 & -1.090368 & 0.020815 \\
\hline & & $\mathrm{H}$ & -2.628576 & -1.106643 & 1.883178 \\
\hline & & $\mathrm{H}$ & -2.541988 & 0.644678 & 1.602292 \\
\hline & & $\mathrm{H}$ & -1.882393 & -1.507149 & -4.045357 \\
\hline & & $\mathrm{H}$ & -3.241747 & -1.590688 & -2.911112 \\
\hline & & $\mathrm{H}$ & 1.914131 & 1.162210 & 2.597136 \\
\hline & & $\mathrm{C}$ & -3.358814 & -4.583610 & -2.924030 \\
\hline & & $\mathrm{H}$ & -2.235553 & -4.815420 & -4.783081 \\
\hline & & $\mathrm{C}$ & -3.191550 & -4.443618 & -4.417866 \\
\hline & & $\mathrm{O}$ & -4.762677 & -5.654009 & -3.490023 \\
\hline & & $\mathrm{C}$ & -4.373064 & -5.365813 & -4.694527 \\
\hline & & $\mathrm{O}$ & -4.831240 & -5.742890 & -5.754621 \\
\hline & & $\mathrm{H}$ & 2.808480 & -0.798273 & 0.631902 \\
\hline & & $\mathrm{H}$ & -3.950262 & -3.884798 & -2.354739 \\
\hline & & $\mathrm{H}$ & -2.834651 & -5.353346 & -2.383417 \\
\hline & & $\mathrm{H}$ & -3.337105 & -3.426708 & -4.781549 \\
\hline \multirow[t]{12}{*}{ Reactant state } & -1671.108 & $\mathrm{C}$ & -1.407287 & -1.318594 & 1.210110 \\
\hline & & $\mathrm{N}$ & -0.768908 & -0.677544 & 2.225979 \\
\hline & & $\mathrm{C}$ & 0.049447 & 0.439502 & 1.983503 \\
\hline & & $\mathrm{N}$ & 0.190985 & 0.839145 & 0.682769 \\
\hline & & $\mathrm{C}$ & -0.431912 & 0.209050 & -0.274455 \\
\hline & & $\mathrm{C}$ & -1.286528 & -0.927076 & -0.080957 \\
\hline & & $\mathrm{O}$ & 0.591501 & 0.995825 & 2.924170 \\
\hline & & $\mathrm{O}$ & -0.289135 & 0.612728 & -1.526141 \\
\hline & & $\mathrm{C}$ & 1.143031 & 3.942519 & 1.597915 \\
\hline & & $\mathrm{H}$ & 3.265075 & 3.711469 & 0.908309 \\
\hline & & $\mathrm{C}$ & 2.325298 & 3.170417 & 1.003374 \\
\hline & & $\mathrm{C}$ & 1.469082 & 3.190435 & -0.243615 \\
\hline
\end{tabular}




\begin{tabular}{cccc}
\hline $\mathrm{O}$ & 1.515641 & 2.749746 & -1.355270 \\
$\mathrm{H}$ & 2.467894 & 2.167683 & 1.400428 \\
$\mathrm{O}$ & 0.473897 & 3.918469 & 0.293949 \\
$\mathrm{H}$ & 1.328261 & 4.970158 & 1.903281 \\
$\mathrm{H}$ & 0.565145 & 3.382288 & 2.328345 \\
$\mathrm{C}$ & -0.912255 & -1.097713 & 3.615097 \\
$\mathrm{H}$ & 0.070741 & -1.298541 & 4.039813 \\
$\mathrm{H}$ & -1.526669 & -1.995753 & 3.648738 \\
$\mathrm{H}$ & -1.378421 & -0.302452 & 4.197220 \\
$\mathrm{H}$ & 0.311124 & 1.385912 & -1.563087 \\
$\mathrm{C}$ & -1.965014 & -1.596610 & -1.237451 \\
$\mathrm{H}$ & -1.231513 & -1.964980 & -1.957529 \\
$\mathrm{H}$ & -2.611693 & -0.894540 & -1.767548 \\
$\mathrm{H}$ & -2.569604 & -2.437218 & -0.894089 \\
$\mathrm{H}$ & -2.020422 & -2.166066 & 1.495979 \\
\hline
\end{tabular}


Table S20. Absolute energies and Cartesian atomic coordinates for the acylation reaction of glutathione at the M06-2X/6-311++G(d,p) level of theory

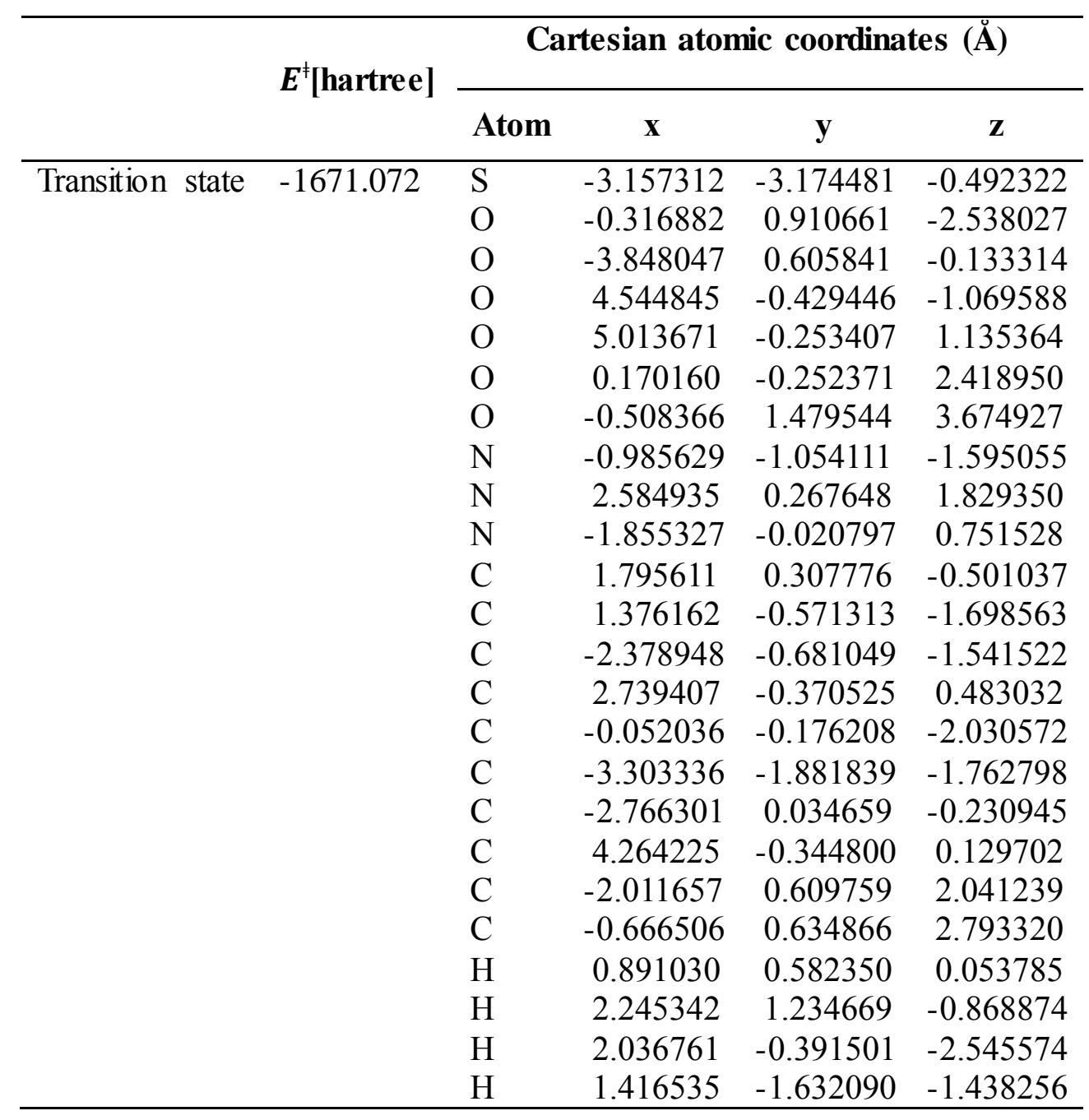




\begin{tabular}{|c|c|c|c|c|c|}
\hline & & $\mathrm{H}$ & -2.572612 & 0.054001 & -2.328454 \\
\hline & & $\mathrm{H}$ & -0.691525 & -2.022177 & -1.299903 \\
\hline & & $\mathrm{H}$ & 3.418696 & -0.020212 & 2.354537 \\
\hline & & $\mathrm{H}$ & 1.597115 & 0.036328 & 2.264307 \\
\hline & & $\mathrm{H}$ & -1.035223 & -0.603423 & 0.639208 \\
\hline & & $\mathrm{H}$ & -2.741925 & 0.072713 & 2.659839 \\
\hline & & $\mathrm{H}$ & -2.376908 & 1.629796 & 1.918724 \\
\hline & & $\mathrm{H}$ & -3.083918 & -2.365479 & -2.718167 \\
\hline & & $\mathrm{H}$ & -4.334630 & -1.529011 & -1.760471 \\
\hline & & $\mathrm{H}$ & 2.651628 & 1.279824 & 1.726708 \\
\hline & & $\mathrm{H}$ & 2.435286 & -1.413177 & 0.620398 \\
\hline & & $\mathrm{O}$ & -2.265521 & -4.599469 & -2.602770 \\
\hline & & $\mathrm{C}$ & -2.039842 & -4.389673 & -1.433679 \\
\hline & & $\mathrm{O}$ & -0.305430 & -3.581478 & -1.125440 \\
\hline & & $\mathrm{H}$ & -1.017425 & -6.204960 & -0.992223 \\
\hline & & $\mathrm{C}$ & -1.418343 & -5.364093 & -0.421639 \\
\hline & & $\mathrm{C}$ & -0.319848 & -4.388657 & 0.010364 \\
\hline & & $\mathrm{H}$ & 0.649242 & -4.871832 & 0.206973 \\
\hline & & $\mathrm{H}$ & -2.126602 & -5.721070 & 0.334161 \\
\hline & & $\mathrm{H}$ & -0.614083 & -3.842176 & 0.921827 \\
\hline \multirow[t]{12}{*}{ Reactant state } & -1671.108 & $\mathrm{~S}$ & -1.373821 & -2.455622 & -2.382194 \\
\hline & & $\mathrm{O}$ & 0.115202 & 2.543497 & -1.882737 \\
\hline & & $\mathrm{O}$ & -3.844057 & 0.682901 & -1.397472 \\
\hline & & $\mathrm{O}$ & 3.902036 & -2.017124 & -0.007635 \\
\hline & & $\mathrm{O}$ & 2.293175 & -3.279142 & 0.950045 \\
\hline & & $\mathrm{O}$ & -0.594774 & 0.101253 & 2.110944 \\
\hline & & $\mathrm{O}$ & -2.223695 & 0.701486 & 3.539927 \\
\hline & & $\mathrm{N}$ & -0.301830 & 0.326980 & -2.125106 \\
\hline & & $\mathrm{N}$ & 0.330766 & -1.713327 & 0.344734 \\
\hline & & $\mathrm{N}$ & -2.039035 & 0.633005 & -0.030751 \\
\hline & & $\mathrm{C}$ & 1.989338 & 0.158662 & -0.118478 \\
\hline & & $\mathrm{C}$ & 1.906291 & 1.000660 & -1.408363 \\
\hline
\end{tabular}




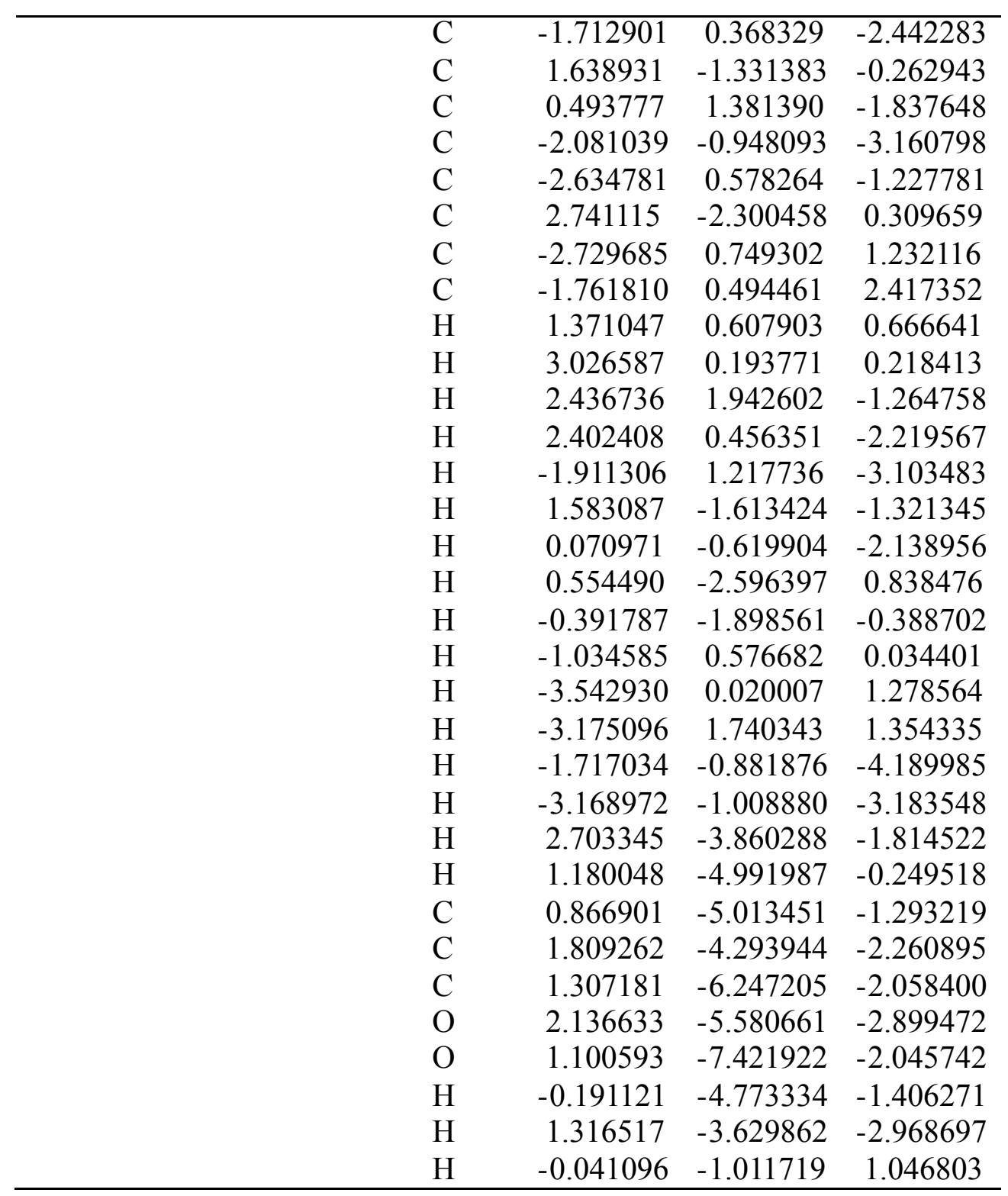


